NUREG/CR-1735

PNL-3765

Addendum 1

\title{
Experiment Operations Plan for a Loss-of-Coolant Accident Simulation in the National Research \\ Universal Reactor
}

Materials Tests 1 and 2

Prepared by G. E. Russcher, C. L. Wilson, R. K. Marshall, L. L. King,

L. J. Parchen, J. P. Pilger, G. M. Hesson, C. L. Mohr

Pacific Northwest Laboratory

Operated by

Battelle Memorial Institute

Prepared for

U.S. Nuclear Regulatory

Commission 


\section{NOTICE}

This report was prepared as an account of work sponsored by an agency of the United States Government. Neither the United States Government nor any agency thereof, or any of their employees, makes any warranty, expressed or implied, or assumes any legal liability or responsibility for any third party's use, or the results of such use, of any information, apparatus product or process disclosed in this report, or represents that its use by such third party would not infringe privately owned rights.

Available from

GPO Sales Program

Division of Technical Information and Document Control

U. S. Nuclear Regulatory Commission

Washington, D. C. 20555

Printed copy price: $\$ 3.00$

and

Nätional Technical Information Service

Springfield, Virginia 22161 
NUREG/CR-1735

PNL-3765

Addendum 1

R3

\section{Experiment Operations Plan for a Loss-of-Coolant Accident Simulation in the National Research Universal Reactor}

Materials Tests 1 and 2

Manuscript Completed: March 1981

Date Published: September 1981

Prepared by

G. E. Russcher, C. L. Wilson, A. K. Marshall, L. L. King,

L. J. Parchen, J. P. Pilger, G. M. Hesson, C. L. Mohr

Pacific Northwest Laboratory

Richland, WA 99352

Prepared for

Division of Accident Evaluation

Office of Nuclear Regulatory Research

U.S. Nuclear Regulatory Commission

Washington, D.C. 20555

NRC FIN B2277 


\author{
.
}

\author{
.
}




\section{ABSTRACT}

A Loss of Coolant Accident (LOCA) simuiation program is evaluating the thermal-hydraulic and mechanical effects of LOCA conditions on pressurized water reactor test fuel bundles. This experiment operation plan for the second and third experiments of the program will provide peak fuel cladding temperatures of up to $1172 \mathrm{~K}\left(1650^{\circ} \mathrm{F}\right)$ and $1061 \mathrm{~K}\left(1450^{\circ}\right)$ respectively, for a long enough time to cause test fuel cladding deformation and rupture in both.

Reflood coolant delay times and the reflooding rates for the experiments were selected from thermal-hydraulic data measured in the National Research Universal (NRU) reactor facilities and test train assembly during the first experiment. 

Pacific Northwest Laboratory (PNL) is conducting a series of experiments in the U-2 loop of the National Research Universal (NRU) Reactor at Chalk River, Ontario, Canada, to evaluate the consequences of a simulated loss-of-coolant accident (LOCA) in a pressurized water reactor (PWR).

The second and third experiments of the LOCA simulation program being conducted by PNL are designed to evaluate the scenario and effects of PWR fue 1 cladding failure. The initial prototypic thermal-hydraulic (PTH) experiment of this program did not exhibit fuel failure because fuel rods were unpressurized. However, cladding deformation materials tests (MT) will rupture test fuel rods because they are pressurized with He to simulate beginning-of-life PWR fuel. Materials test trains MT-1 and MT-2 will probably incur fuel failure at peak fuel cladding temperatures determined by the thermal-hydraulic conditions characterized in the PTH experiment. However, even if test fuel rupture does not occur, the experiment $w i l l$ be terminated after reflooding coolant has quenched the fuel bundle.

Pacific Northwest Laboratory will provide the materials test trajns, the test fuel assembiies, data acquisition, post irradiation examination, data reduction and analysis, and the management of the LOCA simulation program. Atomic Energy of Canada, Ltd., (AECL) will provide the test facilities, the irradiation space in the NRU Reactor, and operation and support of the facilities. 



\section{CONTENTS}

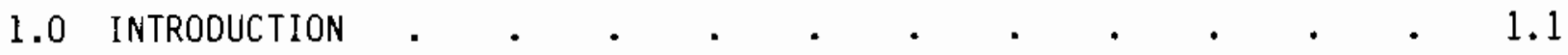

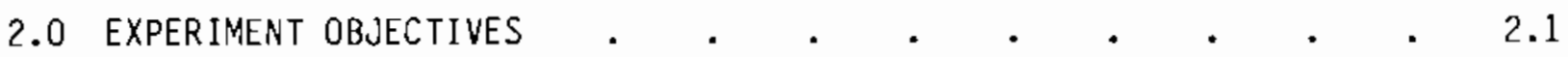

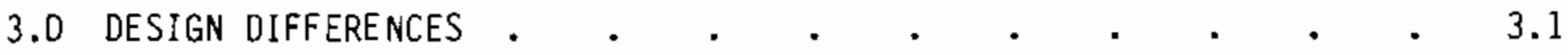

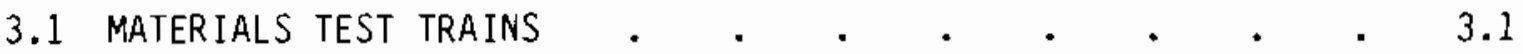

3.2 LOOP FACILITIES AND THE LOOP CONTROL SYSTEM • $\quad$ • $\quad$ • 3.9

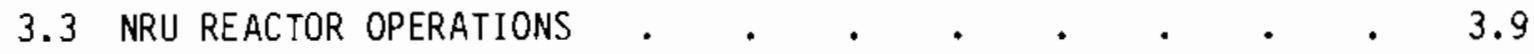

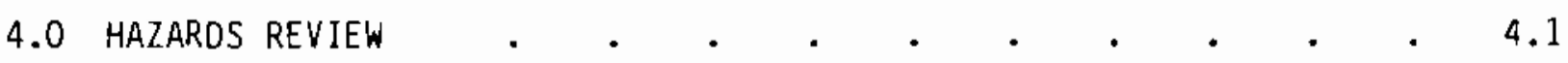

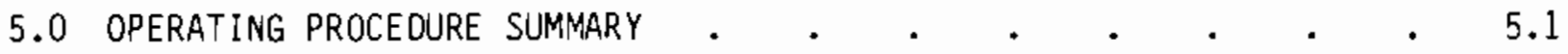

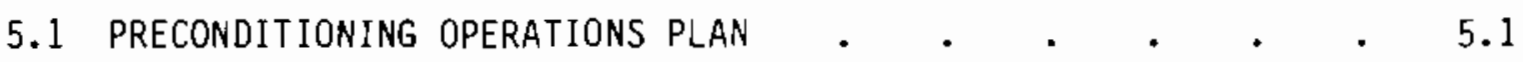

5.1.1 Piping Configuration . . . . . . . . 5.1

5.1 .2 Loop Facilities Preparations $\quad$. $\quad . \quad$. 5.1

5.1.3 NRU Reactor Preparation . . . . . . . 5.1

5.1.4 OACS Computer Preparation . $\quad$. $\quad$. $\quad . \quad$. 5.1

5.1.5 Preconditioning Operating Procedure . . . $\quad$. 5.3

5.2 REFLOOD FLOW, TEST PRETRANSIENT AND

TRANSIENT OPERATIONS PLAN $. \quad . \quad . \quad . \quad . \quad . \quad$. 5.5

5.2.1 Piping Configuration . . . . . . . . 5.5

5.2.2 Loop System Preparation . . . . . . . . 5.5

5.2.3 NRU Reactor Preparations . • • • . . . . 5.7

5.2.4 DACS Computer Preparation . $\quad$ • $\quad . \quad$. 5.9

5.2.5 Reflood Flow Test Operating Procedure . . $\quad$. 5.9

5.2.6 Pretransient Operating Procedure . . . . $\quad$. 5.11

5.2.7 Transient Dperating Procedure . $\quad$. . . . 5.11 


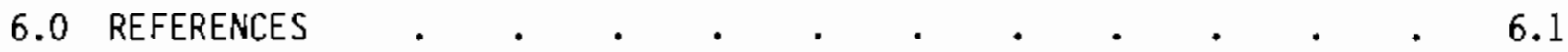
APPENDIX A - REACTOR LOADING FOR BATTELLE LOCA SIMULATION TESTS . . A.1

\section{FIGURES}

3.1 Measured Temperature Distributions and NRU Trip Setpoints . . 3.8

3.2 MT Experiment and NRU Operations Schematic . . . . . . . 3.11

\section{TABLES}

3.1 Materials Test Train Design Differences . . . . . . 3.2

3.2 Instrumented Data Summary . . . . . . . . . . . 3.3

3.3 Trip Setpoint Criteria for Operating and Non-Functional
Fuel Cladding Thermocouples . . . . . . . . . 3.7

4.1 Expected Radioactive Release from Eleven Test
Fuel Rod Ruptures . . . . . . 4.2

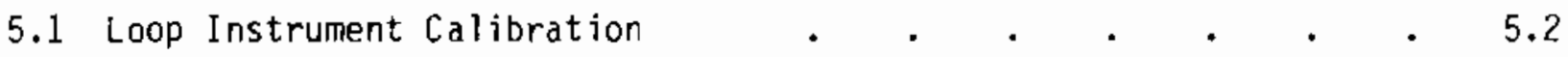

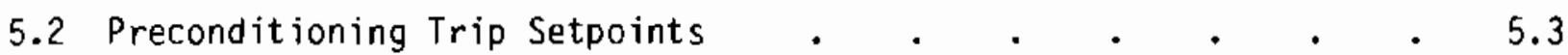

5.3 Preconditioning Trip Setpoints for the U-2 Loop . . . . 5.3

5.4 Pretransient And Transient Trip Setpoints . . . . . . . 5.6

5.5 Cladding High Temperature Trip Sensors . . . . . . . . 5.7

5.6 Parameters for Transient and Reflood Flow Tests
for MT-1 and MT-2 . . . . . . . . . . . . . . . . 5.8 


\section{$1.0 \quad$ INTRODUCTION}

Pacific Northwest Laboratory $(P N L)^{(a)}$ is conducting an experimental program for the Nuclear Regulatory Commission (NRC), Division of Reactor Safety Research, Fuel Behavior Branch, to evaluate the consequences of a hypothetical loss-of-coolant accident (LOCA) in a pressurized water reactor (PWR). Pacific Northwest Laboratory will provide the materials test trains, the test fuel assemblies, post irradiation examination of the test trains, data reduction and analysis, and management of the LOCA simulation program. Atomic Energy of Canada, Ltd. will provide test facilities and irradiation space in the Canadian Nationa] Research Universal (NRU) Reactor at the Chalk River Nuclear Laboratories (CRNL), Chalk River, Ontario.

A LOCA in a commercial PWR involves four major, distinct phases: blowdown, heatup, reflood, and quench. Each of these phases involves a pathdependent process that is a function of 1) the type of event that initiated the accident and 2) the reactor operating conditions at the time the LOCA was initiated. During the most damaging LOCA phases, heatup and reflood, the highest fuel cladding temperatures are reached, with the maximum pressure differential occurring across the fuel cladding wall. The pressurized cladding will rupture when the critical pressure is reached, releasing fission products possibily during the heatup phase.

Two cladding deformation materials tests (MT-1 and MT-2), that are the subject of this addendum report, will characterize PWR fuel rod rupture due to prepressurization at beginning of life. These experiments will attain peak cladding temperatures of up to $1172 \mathrm{~K}\left(1650^{\circ} \mathrm{F}\right)$ for MT-1 and up to $1061 \mathrm{~K}$ $\left(1450^{\circ} \mathrm{F}\right)$ for MT-2. Operating conditions necessary to achieve the desired peak fuel cladding temperatures (and the predicted fuel cladding rupture) were developed in the prototypic thermal-hydraulic (PTH) experiment.

The reference (C. L. Mohr et a1. 1980) Safety Analys is Report (SAR) provides a description of the LOCA simulation major safety issues and an overview of the experiments that were planned for the program. These safety issues

(a) Operated by Battelle Memorial Institute. 
were concurred with and further reported (D. J. Axford, I.C. Martin, and S. J. McAuley 1980). Because the experiment safety issues have been adequately analyzed, reviewed and reported, no further safety analyses are planned for these experiments. In this operations plan addendum, any changes to the test train or experiment operation are referenced to the appropriate safety document. Previous data or discussions are not repeated here. However, information is presented (in the Hazards Review, section 4.0) to show that differences between the MT and the PTH experiments are either insignificant safety issues or they are enveloped by the safety ancilyses prisented before.

The Experiment Operations Pian (EOP) (G. E. Russcher et al. 1980) provides a detailed summary of the PTH experiment, and also serves as the techrical basis for the subsequent MT experiments thas are discussed here. Most. design aspects of their test train and test fuel assemblies are identica?. Similarly, most planned operations are th s ane. Consequently, the pertinent discussions and information found in the referunce EOD are nct reproduced here, just referenced.

This addendum to the reforence EOP is desigried to present orily the new or different information that is needed to evaluate ine materials test trains (MT-1 and MT-2) and their planned perfornanca, to evilute exiectad operat: ais of the data acquisition and control systen: (DACS) ans the loop coltrol system (1CS), and to plan the necessary NRij and oup iaility operations. This emphasis on the differences between previous anc cuirent tust. trains and experinent. operations means that many topics are not discussed asain, ratner just refurenced to the document where they vere cover:c.

In addition, this report contains an Optrating procedure Summary (section 5.0) tnat shows the sequence of activitits that PiL and CRNL operations persconnel are expected to perform ior thr Mit ex,iceritients. 


\subsection{EXPER IMENT OBJECTIVES}

The primary objective is to characterize fuel cladding rupture during two LOCA simulation experiments that result in peak fuel cladding temperatures as high as $1172 \mathrm{~K}\left(1650^{\circ} \mathrm{F}\right)$ and $1061 \mathrm{~K}\left(1450^{\circ} \mathrm{F}\right)$, respectively. These experiments, MT-1 and MT-2, will use two different, but nearly identical, materials test trains MT-1 and MT-2. Each CMD transient phase is designed to conclude with cladding rupture of as many as 11 test fue r rods. The operating conditions necessary to achieve the desired peak fuel cladding temperatures (and rupture) are derived from the PTH experiment that characterized system thermal-hydraulic response during LOCA simulations.

A secondary objective is to determine the fuel rod rupture time and location during the simulated LOCA experiments. Current fuel rupture models will be used to predict fuel cladding rupture and select experiment operating conditions as well as possible. These predictions will also be used to plan post irradiation examinations and to compare predicted with measured experiment results.

Another experiment objective is to evaluate the thermal-hydraulic effects of the fuel cladding rupture. Reflooding hydraulic characteristics of the test train assemblies will be determined before and after the simulated LOCA transients. Thermal characteristics will also be measured during the transient to evaluate the cooldown of cladding due to lift off or separation at the fuel/ cladding interface. 



\subsection{DESIGN DIFFERENCES}

\subsection{MATERIALS TEST TRAINS}

The basic difference between the test train used for the PTH experiment and the MT experiment is that the 11 MT test fue 1 rods are pressurized with He to provide internal cladding stress that simulates PWR prepressurization at beginning of life. This pressurization is designed to cause simulated LOCA fue 1 rod rupture while minimizing the radioactive fission product inventory. The design differences of the materials test trains are summarized in Table 3.1. Only the 11 test fuel rods are pressurized with He during fabrication. The remaining 20 guard fuel rods are unpressurized for all simulated LOCA experiments.

Instrumentation changes are also planned that will meet the need for additional data during the CMD experiments. All of the data to be recorded by DACS are summarized in Table 3.2. The DACS number, name and the sensor location are identified for each. Three additional data types are presented here that were not available for the PTH experiment.

1. Each test fuel rod has a pressure transducer (either continuous or a switch type) attached to the top end cap. Continuous pressure transducers will monitor the internal pressure for test fuel rods $3 \mathrm{C}$ and $5 C$, but pressure switches will identify the rupture time for the other fuel rods.

2. Interior fuel cladding TCS are attached to the cladding to monitor its temperature without interference from fuel pellet chips or unintentional TC relocation. In addition, several of these TCS (sensors 91, 94, 97, 98, 100,106,108, 117, 120,123,126, and 137) are now axially relocated to regions of higher temperature (lower instrument levels).

3. Guard fue 1 rod cladding TCS selected for NRU trip circuitry are compared with PTH data. They (DACS pseudo sensors 305, 308, 315) are identified on Figure 3.1, together with an axial distribution of cladding temperatures during the PTH-125 transient that produced a 
peak cladding temperature of about $1255 \mathrm{~K}\left(1800^{\circ} \mathrm{F}\right)$. Example temperature distributions from PTH-125 data are shown because they provide instrument tube and average fuel cladding temperatures for comparison with trip setpoint temperatures of the PTH experiment. It is evident that guard fuel cladding temperatures at instrument level 13 were consistently higher than at level 17, as typified on Figure 3.1. Peak fuel cladding temperatures occurred between levels 13 and 15 rather than between levels 15 and 17 . Consequently, the fuel cladding maximum trip setpoint temperature [1361K $\left.\left(1990^{\circ} \mathrm{F}\right)\right]$, calculated in Appendix $D$ of the reference EOP, will be applied to sensors on levels 13 and 15 for the MT experiments. Similarly, because of the lower temperatures measured at level 17 in the PTH experiment, a lower trip setpoint temperature [1333K $\left.\left(1940^{\circ} \mathrm{F}\right)\right]$ will be used for the sensors on level 17. The tally of the trip setpoint safety margins, uncertainties and error margins developed in the reference EOP requires no modifications or further justification. The same is true for the pressure tube and hanger tube setpoint temperatures. During the most severe transients, measured temperatures were at least $28 \mathrm{~K}\left(50^{\circ} \mathrm{F}\right)$ less than the acceptable temperatures reported in the reference SAR (C. L. Mohr et al. 1980). Consequently, the same pressure tube temperature limits and hanger tube trip setpoint temperature limits used in the PTH experiment (G. E. Russcher et al. 1980 - Appendix D) will be used in the MT experiments. See Table 3.3 for a summary of fuel cladding high temperature trip sensors and trip setpoints under various operating conditions.

TABLE 3.1. Materials Test Train Design Differences

\begin{tabular}{|c|c|c|}
\hline $\begin{array}{c}\text { Simulated LOCA } \\
\text { Experiment } \\
\end{array}$ & $\begin{array}{c}\text { Peak Cladding } \\
\text { Temperature } \\
\end{array}$ & $\begin{array}{r}\text { Test Fuel Rod } \\
\text { Internal Pressure, STP }\end{array}$ \\
\hline$M T-1$ & $1172 \mathrm{~K}\left(1650^{\circ} \mathrm{F}\right)$ & $-3.1 \mathrm{MPa}$ (450 psia) \\
\hline$M T-2$ & $1061 \times\left(1450^{\circ} \mathrm{F}\right)$ & $-3.4 \mathrm{MPa}(500 \mathrm{psia})$ \\
\hline
\end{tabular}


TABLE 3.2. Instrumented Data Summary

\begin{tabular}{|c|c|c|c|c|c|c|c|}
\hline \multirow[b]{2}{*}{ No. } & \multirow[b]{2}{*}{ DACS Name } & \multicolumn{2}{|c|}{ Location } & \multirow[b]{2}{*}{ No. } & \multirow[b]{2}{*}{ DACS Name } & \multicolumn{2}{|c|}{ Location } \\
\hline & & in. & Component & & & in. & Component \\
\hline $\begin{array}{l}1 \\
2 \\
3 \\
4\end{array}$ & $\begin{array}{l}T C-1-4 D-I N \\
T C-1-6 A-I N \\
T C-1-1 F-I N \\
T C-2-6 F-S-C\end{array}$ & $\begin{array}{r}-0.95 \\
-0.95 \\
-0.95 \\
8.03\end{array}$ & $\begin{array}{l}\text { MT }(a) \\
\text { MT } \\
\text { MT } \\
\text { MT }\end{array}$ & $\begin{array}{l}37 \\
38 \\
39 \\
40\end{array}$ & $\begin{array}{l}\text { TC-9-1F-S-C } \\
\text { TC-10-4D-SP-4 } \\
\text { TC-10-6A-S-C } \\
\text { TC-10-6F-S-C }\end{array}$ & $\begin{array}{l}48.32 \\
55.28 \\
55.28 \\
55.28\end{array}$ & $\begin{array}{l}\text { MT } \\
\text { MT }(c) \\
\text { MT } \\
\text { MT }\end{array}$ \\
\hline $\begin{array}{l}5 \\
6 \\
7 \\
8\end{array}$ & $\begin{array}{l}\text { TC-2-1A-S-C } \\
\text { UNDEF INED } \\
\text { TC-3-5B-OR-1 } \\
\text { TC-3-5E-OR-2 }\end{array}$ & $\begin{array}{r}8.03 \\
13.28 \\
13.28\end{array}$ & $\begin{array}{l}\text { MT (b) } \\
\text { MT } \\
\text { MT }\end{array}$ & $\begin{array}{l}41 \\
42 \\
43 \\
44\end{array}$ & $\begin{array}{l}\text { TC-10-1F-S-C } \\
\text { TC-10-1A-S-C } \\
\text { TC-11-6C--OR-4 } \\
\text { TC-11-4F-0R-1 }\end{array}$ & $\begin{array}{l}55.28 \\
55.28 \\
60.53 \\
60.53\end{array}$ & $\begin{array}{l}\text { MT } \\
\text { MT } \\
\text { MT } \\
\text { MT }\end{array}$ \\
\hline $\begin{array}{r}9 \\
10 \\
11 \\
12\end{array}$ & $\begin{array}{l}T C-3-2 E-0 R-3 \\
T C-3-2 B-0 R-4 \\
T C-3-6 A-S-C \\
T C-3-1 F-S-C\end{array}$ & $\begin{array}{l}13.28 \\
13.28 \\
13.28 \\
13.28\end{array}$ & $\begin{array}{l}\text { MT } \\
\text { MT } \\
\text { MT } \\
\text { MT }\end{array}$ & $\begin{array}{l}45 \\
46 \\
47 \\
48\end{array}$ & $\begin{array}{l}\text { TC-11-1D-OR-2 } \\
\text { TC-11-3A-0R-3 } \\
\text { TC-11-6F-S-C } \\
\text { TC-11-1A-S-C }\end{array}$ & $\begin{array}{l}60.53 \\
60.53 \\
60.53 \\
60.53\end{array}$ & $\begin{array}{l}\text { MT } \\
\text { MT } \\
\text { MT } \\
\text { MT }\end{array}$ \\
\hline $\begin{array}{l}13 \\
14 \\
15 \\
16\end{array}$ & $\begin{array}{l}\text { TC-4-6F-S-C } \\
\text { TC-4-1A-S-C } \\
\text { TC-5-6A-S-C } \\
\text { TC-5-IF-S-C }\end{array}$ & $\begin{array}{l}18.53 \\
18.53 \\
27.32 \\
27.32\end{array}$ & $\begin{array}{l}\text { MT } \\
\text { MT } \\
\text { MT } \\
\text { MT }\end{array}$ & $\begin{array}{l}49 \\
50 \\
51 \\
52\end{array}$ & $\begin{array}{l}\text { UNDEF INED } \\
\text { TC-12-5B-SP-3 } \\
\text { TC-12-5E-SP-4 } \\
\text { TC-12-2E-SP-1 }\end{array}$ & $\begin{array}{l}68.32 \\
68.32 \\
68.32\end{array}$ & $\begin{array}{l}\text { (b) } \\
\text { MT } \\
\text { MT } \\
\text { MT }\end{array}$ \\
\hline $\begin{array}{l}17 \\
18 \\
19 \\
20\end{array}$ & $\begin{array}{l}\text { UNDEF INED } \\
\text { TC-6-6A-S-C } \\
\text { TC-6-6F-S-C } \\
\text { TC-6-1F-S-C }\end{array}$ & $\begin{array}{l}34.28 \\
34.28 \\
34.28\end{array}$ & $\begin{array}{l}\text { (b) } \\
\text { MT } \\
\text { MT } \\
\text { MT }\end{array}$ & $\begin{array}{l}53 \\
54 \\
55 \\
56\end{array}$ & $\begin{array}{l}\text { TC-12-28-SP-2 } \\
\text { TC-13-4D-SP-4 } \\
\text { TC-13-3B-IR-2 } \\
\text { TC-13-6C-IR-2 }\end{array}$ & $\begin{array}{l}68.32 \\
76.28 \\
76.28 \\
76.28\end{array}$ & $\begin{array}{l}\text { MT } \\
\text { MT (c) } \\
\text { MT } \\
\text { MT (d) }\end{array}$ \\
\hline $\begin{array}{l}21 \\
22 \\
23 \\
24\end{array}$ & $\begin{array}{l}\text { TC-6-1A-S-C } \\
\text { TC-7-6C-OR-4 } \\
\text { TC-7-4F-0R-1 } \\
\text { TC-7-1D-0R-2 }\end{array}$ & $\begin{array}{l}34.28 \\
39.53 \\
39.53 \\
39.53\end{array}$ & $\begin{array}{l}\text { MT } \\
\text { MT } \\
\text { MT } \\
\text { MT }\end{array}$ & $\begin{array}{l}57 \\
58 \\
59 \\
60\end{array}$ & $\begin{array}{l}\text { TC-13-4F-IR-4 } \\
\text { TC-13-1D-1R-2 } \\
\text { TC-13-3A-IR-4 } \\
\text { TC-13-6A-S-C }\end{array}$ & $\begin{array}{l}76.28 \\
76.28 \\
76.28 \\
76.28\end{array}$ & $\begin{array}{l}\operatorname{MT}(d) \\
\operatorname{MT}(d) \\
\operatorname{MT}(d) \\
M T\end{array}$ \\
\hline $\begin{array}{l}25 \\
26 \\
27 \\
28\end{array}$ & $\begin{array}{l}T C-7-3 A-0 R-3 \\
T C-8-5 B-0 R-1 \\
T C-8-2 E-0 R-3 \\
T C-8-5 E-0 R-2\end{array}$ & $\begin{array}{l}39.53 \\
42.24 \\
42.24 \\
42.24\end{array}$ & $\begin{array}{l}\text { MT } \\
\text { MT } \\
\text { MT } \\
\text { MT }\end{array}$ & $\begin{array}{l}61 \\
62 \\
63 \\
64\end{array}$ & $\begin{array}{l}\text { TC-13-6F-S-C } \\
\text { TC-13-1F-S-C } \\
\text { TC-13-1A-S-C } \\
\text { TC-14-4D-SP-4 }\end{array}$ & $\begin{array}{l}76.28 \\
76.28 \\
76.28 \\
89.32\end{array}$ & $\begin{array}{l}\text { MT } \\
\text { MT } \\
\text { MT } \\
\text { MT (c) }\end{array}$ \\
\hline $\begin{array}{l}29 \\
30 \\
31 \\
32\end{array}$ & $\begin{array}{l}\text { TC-8-2B-0R-4 } \\
\text { TC-8-6F-S-C } \\
\text { TC-8-1A-S-C } \\
\text { TC-9-5B-0R-1 }\end{array}$ & $\begin{array}{l}42.24 \\
42.24 \\
42.24 \\
48.32\end{array}$ & $\begin{array}{l}\text { MT } \\
\text { MT } \\
\text { MT } \\
\text { MT }\end{array}$ & $\begin{array}{l}65 \\
66 \\
67 \\
68\end{array}$ & $\begin{array}{l}\mathrm{TC}-14-5 \mathrm{~B}-\mathrm{SP}-3 \\
\mathrm{TC}-14-5 \mathrm{E}-\mathrm{SP}-4 \\
\mathrm{TC}-14-2 \mathrm{E}-\mathrm{SP}-1 \\
\mathrm{TC}-14-2 \mathrm{~B}-\mathrm{SP}-2\end{array}$ & $\begin{array}{l}89.32 \\
89.32 \\
89.32 \\
89.32\end{array}$ & $\begin{array}{l}\text { MT } \\
\text { MT } \\
\text { MT } \\
\text { MT }\end{array}$ \\
\hline $\begin{array}{l}33 \\
34 \\
35 \\
36\end{array}$ & $\begin{array}{l}\text { TC-9-5E-OR-2 } \\
\text { TC-9-2E-0R-3 } \\
\text { TC-9-2B-0R-4 } \\
\text { TC-9-6A-S-C }\end{array}$ & $\begin{array}{l}48.32 \\
48.32 \\
48.32 \\
48.32\end{array}$ & $\begin{array}{l}\text { MT } \\
\text { MT } \\
\text { MT } \\
\text { MT }\end{array}$ & $\begin{array}{l}69 \\
70 \\
71 \\
72\end{array}$ & $\begin{array}{l}\text { TC-15-4D-SP-4 } \\
\text { TC-15-38-IR-4 } \\
\text { TC-15-6C-IR-4 } \\
\text { TC-15-4F-IR-2 }\end{array}$ & $\begin{array}{l}97.28 \\
97.28 \\
97.28 \\
97.28\end{array}$ & $\begin{array}{l}\operatorname{MT}(c) \\
\operatorname{MT} \\
\operatorname{MT}(e) \\
\operatorname{MT}(e)\end{array}$ \\
\hline
\end{tabular}




\begin{tabular}{|c|c|c|c|c|c|c|c|}
\hline \multirow[b]{2}{*}{ No. } & \multirow[b]{2}{*}{ DACS Name } & \multicolumn{2}{|c|}{ Location } & \multirow[b]{2}{*}{ No. } & \multirow[b]{2}{*}{ DACS Name } & \multicolumn{2}{|c|}{ Location } \\
\hline & & in. & Component & & & in. & Component \\
\hline $\begin{array}{l}73 \\
74 \\
75 \\
76\end{array}$ & $\begin{array}{l}\text { TC-15-1D-IR-4 } \\
\text { TC-15-3A-IR-2 } \\
\text { TC-15-6A-S-C } \\
\text { TC-15-6F-S-C }\end{array}$ & $\begin{array}{l}97.28 \\
97.28 \\
97.28 \\
97.28\end{array}$ & $\begin{array}{l}M T(e) \\
M T(e) \\
M T \\
M T\end{array}$ & $\begin{array}{l}109 \\
110 \\
111 \\
112\end{array}$ & $\begin{array}{l}\text { TC-17-2B-IR-4 } \\
\text { TC-17-6A-S-C } \\
\text { TC-17-6F-S-C } \\
\text { TC-17-1F-S-C }\end{array}$ & $\begin{array}{l}118.28 \\
118.28 \\
118.28 \\
118.28\end{array}$ & $\begin{array}{l}M T(f) \\
M T \\
M T \\
M T\end{array}$ \\
\hline $\begin{array}{l}77 \\
78 \\
79 \\
80\end{array}$ & $\begin{array}{l}\text { TC-15-1F-S-C } \\
\text { TC-15-1A-S-C } \\
\text { TC-16-4D-SP-4 } \\
\text { TC-16-5B-SP-3 }\end{array}$ & $\begin{array}{r}97.28 \\
97.28 \\
110.32 \\
110.32\end{array}$ & $\begin{array}{l}\text { MT } \\
M T \\
M T(c) \\
M T\end{array}$ & $\begin{array}{l}113 \\
114 \\
115 \\
116\end{array}$ & $\begin{array}{l}\text { TC-17-1A-S-C } \\
\text { UNDEF INED } \\
\text { TC-18-4B-0R-3 } \\
\text { TC-18-5C-DR-3 }\end{array}$ & $\begin{array}{l}118.28 \\
139.28 \\
139.28\end{array}$ & $\begin{array}{l}\text { MT (b) } \\
\text { MT } \\
\text { MT }\end{array}$ \\
\hline $\begin{array}{l}81 \\
82 \\
83 \\
84\end{array}$ & $\begin{array}{l}\text { TC-16-5E-SP-4 } \\
\text { TC-16-2E-SP-1 } \\
\text { TC-16-2B-SP-2 } \\
\text { TC-16-6A-C-3 }\end{array}$ & $\begin{array}{l}110.32 \\
110.32 \\
110.32 \\
110.32\end{array}$ & $\begin{array}{l}\text { MT } \\
\text { MT } \\
\text { MT } \\
\text { MT }\end{array}$ & $\begin{array}{l}117 \\
118 \\
119 \\
120\end{array}$ & $\begin{array}{l}\text { TC-17-5D-0R-3 } \\
\text { TC-18-4E-0R-4 } \\
\text { TC-18-3E-0R-4 } \\
\text { TC-17-2D-0R-1 }\end{array}$ & $\begin{array}{l}118.28 \\
139.28 \\
139.28 \\
118.28\end{array}$ & $\begin{array}{l}M T(c) \\
M T \\
M T \\
M T(c)\end{array}$ \\
\hline $\begin{array}{l}85 \\
86 \\
87 \\
88\end{array}$ & $\begin{array}{l}\text { TC-16-6F-C-4 } \\
\text { TC-16-1F-C-1 } \\
\text { TC-16-1A-C-2 } \\
\text { TC-17-4C-IR-3 }\end{array}$ & $\begin{array}{l}110.32 \\
110.32 \\
110.32 \\
118.28\end{array}$ & $\begin{array}{l}\text { MT } \\
\text { MT } \\
\text { MT }\end{array}$ & $\begin{array}{l}121 \\
122 \\
123 \\
124\end{array}$ & $\begin{array}{l}\text { TC-18-2C-0R-1 } \\
\text { TC-18-5C-IR-4 } \\
\text { TC-17-5D-IR-4 } \\
\text { TC-18-4E-IR-1 }\end{array}$ & $\begin{array}{l}139.28 \\
139.28 \\
118.28 \\
139.28\end{array}$ & $\begin{array}{l}\text { MT } \\
\text { MT } \\
\text { MT (c) } \\
M T\end{array}$ \\
\hline $\begin{array}{l}89 \\
90 \\
91 \\
92\end{array}$ & $\begin{array}{l}\text { TC-17-4D-SP-4 } \\
\text { TC-17-3D-IR-5 } \\
\text { TC-15-3C-IR-2 } \\
\text { TC-17-4C-IR-C }\end{array}$ & $\begin{array}{r}118.28 \\
118.28 \\
97.28 \\
118.28\end{array}$ & $\begin{array}{l}\operatorname{MT}(c) \\
\operatorname{MT}(c) \\
\operatorname{MT}(c)\end{array}$ & $\begin{array}{l}125 \\
126 \\
127 \\
128\end{array}$ & $\begin{array}{l}\text { TC-18-3E-IR-1 } \\
\text { UNDEF INED } \\
\text { TC-18-2C-IR-2 } \\
\text { TC-18-6A-S-C }\end{array}$ & $\begin{array}{l}139.28 \\
139.28 \\
139.28\end{array}$ & $\begin{array}{l}\text { MT } \\
\text { MT (b) } \\
\text { MT } \\
\text { MT }\end{array}$ \\
\hline $\begin{array}{l}93 \\
94 \\
95 \\
96\end{array}$ & $\begin{array}{l}\text { TC-17-3D-IR-C } \\
\text { TC-15-3C-IR-C } \\
\text { TC-17-4C-IR-1 } \\
\text { TC-17-3D-IR-3 }\end{array}$ & $\begin{array}{r}118.28 \\
97.28 \\
118.28 \\
118.28\end{array}$ & $\begin{array}{l}\text { MT } \\
M T(c) \\
M T \\
\text { MT }\end{array}$ & $\begin{array}{l}129 \\
130 \\
131 \\
132\end{array}$ & $\begin{array}{l}\text { TC-18-6F-S-C } \\
\text { TC-18-1F-S-C } \\
\text { TC-18-1A-S-C } \\
\text { TC-20-0T-1 }\end{array}$ & $\begin{array}{l}139.28 \\
139.28 \\
139.28 \\
167.70\end{array}$ & $\begin{array}{l}\text { MT } \\
\text { MT } \\
\text { MT } \\
\text { MT }\end{array}$ \\
\hline $\begin{array}{r}97 \\
98 \\
99 \\
100\end{array}$ & $\begin{array}{l}\text { TC-15-3C-IR-7 } \\
\text { TC-15-5B-IR-3 } \\
\text { TC-17-5E-IR-4 } \\
\text { TC-15-2E-IR-1 }\end{array}$ & $\begin{array}{r}97.28 \\
97.28 \\
118.28 \\
97.28\end{array}$ & $\begin{array}{l}\operatorname{MT}(c) \\
\operatorname{MT}(c)(e) \\
\operatorname{MT}(f) \\
\operatorname{MT}(c)\langle e\rangle\end{array}$ & $\begin{array}{l}133 \\
134 \\
135 \\
136\end{array}$ & $\begin{array}{l}\text { TC-20-0T-3 } \\
\text { TC-20-0T-2 } \\
\text { TC-21-HT-1 } \\
\text { UNDEF INED }\end{array}$ & $\begin{array}{l}167.70 \\
167.70 \\
187.23\end{array}$ & $\begin{array}{l}\text { MT } \\
\text { MT } \\
\text { MT }\end{array}$ \\
\hline $\begin{array}{l}101 \\
102 \\
103 \\
104\end{array}$ & $\begin{array}{l}\text { TC-17-2B-IR-2 } \\
\text { TC-15-5B-IR-C } \\
\text { TC-17-5E-IR-C } \\
\text { TC-15-2E-IR-C }\end{array}$ & $\begin{array}{r}118.28 \\
97.28 \\
118.28 \\
97.28\end{array}$ & $\begin{array}{l}M T(f) \\
M T(c) \\
M T \\
M T(c)\end{array}$ & $\begin{array}{l}137 \\
138 \\
139 \\
140\end{array}$ & $\begin{array}{l}\text { TC-15-3C-IR-1 } \\
\text { TC-17-3D-IR-4 } \\
\text { UNDEF INED } \\
\text { UNDEF INED }\end{array}$ & $\begin{array}{r}97.28 \\
118.28\end{array}$ & $\begin{array}{l}M T(c) \\
M T\end{array}$ \\
\hline $\begin{array}{l}105 \\
106 \\
107 \\
108\end{array}$ & $\begin{array}{l}\text { TC-17-2B-IR-C } \\
\text { TC-15-5B-IR-1 } \\
\text { TC-17-5E-IR-2 } \\
\text { TC-15-2E-IR-3 }\end{array}$ & $\begin{array}{r}118.28 \\
97.28 \\
118.28 \\
97.28\end{array}$ & $\begin{array}{l}\operatorname{MT} \\
\operatorname{MT}(c)\langle e\rangle \\
M T(f) \\
M T(c)\langle e\rangle\end{array}$ & $\begin{array}{l}141 \\
142 \\
143 \\
144\end{array}$ & $\begin{array}{l}\text { UNDEF INED } \\
\text { SPND-3-6F-S-3 } \\
\text { SPND-3-1A-S-1 } \\
\text { UNDEF INED }\end{array}$ & $\begin{array}{l}13.28 \\
13.28\end{array}$ & $\begin{array}{l}\text { (b) } \\
M T^{M T} \\
\text { (b) }\end{array}$ \\
\hline
\end{tabular}




\begin{tabular}{|c|c|c|c|c|c|c|c|}
\hline \multirow[b]{2}{*}{ No. } & \multirow[b]{2}{*}{ DACS Name } & \multicolumn{2}{|c|}{ Location } & \multirow[b]{2}{*}{ No. } & \multirow[b]{2}{*}{ DACS Name } & \multicolumn{2}{|c|}{ Location } \\
\hline & & in. & Component & & & in. & Component \\
\hline $\begin{array}{l}145 \\
146 \\
147 \\
148\end{array}$ & $\begin{array}{l}\text { SPND-6-6A-S-2 } \\
\text { SPND-6-1F-S-4 } \\
\text { UNDEF INED } \\
\text { SPND-10-6A-S-2 }\end{array}$ & $\begin{array}{l}34.28 \\
34.28 \\
55.28\end{array}$ & $\begin{array}{l}\text { MT } \\
\text { MT } \\
\text { MT }\end{array}$ & $\begin{array}{l}181 \\
182 \\
183 \\
184\end{array}$ & $\begin{array}{l}\text { PS-19-4E-C } \\
\text { PS-19-4E-C-1 } \\
\text { PS-19-3E-C } \\
\text { PS-19-3E-C-1 }\end{array}$ & $\begin{array}{l}157.0 \\
157.0 \\
157.0 \\
157.0\end{array}$ & $\begin{array}{l}\text { MT } \\
\text { MT } \\
\text { MT } \\
\text { MT }\end{array}$ \\
\hline $\begin{array}{l}149 \\
150 \\
151 \\
152\end{array}$ & $\begin{array}{l}\text { SPND-10-6F-S-3 } \\
\text { SPND-10-IF-S-4 } \\
\text { SPND-10-1A-S-1 } \\
\text { SPND-12-1F-C }\end{array}$ & $\begin{array}{l}55.28 \\
55.28 \\
55.28 \\
68.32\end{array}$ & $\begin{array}{l}\text { MT } \\
\text { MT } \\
\text { MT } \\
\text { MT }\end{array}$ & $\begin{array}{l}185 \\
186 \\
187 \\
188 \\
189\end{array}$ & $\begin{array}{l}\text { PS }-19-2 D-C \\
\text { PS-19-2D-C-1 } \\
\text { PS-19-2C-C } \\
\text { PS-19-2C-C-1 } \\
\text { PS -19-3B-C }\end{array}$ & $\begin{array}{l}157.0 \\
157.0 \\
157.0 \\
157.0 \\
157.0\end{array}$ & $\begin{array}{l}\text { MT } \\
\text { MT } \\
\text { MT } \\
\text { MT } \\
\text { MT }\end{array}$ \\
\hline $\begin{array}{l}153 \\
154 \\
155 \\
156\end{array}$ & $\begin{array}{l}\text { L.VOT-19-4D-LL-0 } \\
\text { SPND-13-6A-S-2 } \\
\text { SPND-13-6F-S-3 } \\
\text { SPND-13-1F-S-4 }\end{array}$ & $\begin{array}{r}157.0 \\
76.28 \\
76.28 \\
76.28\end{array}$ & $\begin{array}{l}M T(g) \\
M T \\
M T \\
M T\end{array}$ & $\begin{array}{l}190 \\
191 \\
192\end{array}$ & $\begin{array}{l}P S-19-3 B-C-1 \\
T C-90-H D-1 \\
T C-90-H D-2\end{array}$ & $\begin{array}{l}157.0 \\
336.0 \\
336.0\end{array}$ & $\begin{array}{l}M T \\
H / L-24(i) \\
H / L-24\end{array}$ \\
\hline $\begin{array}{l}157 \\
158 \\
159 \\
160\end{array}$ & $\begin{array}{l}\text { SPND-13-1A-S-1 } \\
\text { UNDEF INED } \\
\text { SPND-15-6A-S-2 } \\
\text { SPND-15-6F-S-3 }\end{array}$ & $\begin{array}{l}76.28 \\
97.28 \\
97.28\end{array}$ & $\begin{array}{l}\text { MT } \\
\text { MT } \\
\text { MT }\end{array}$ & $\begin{array}{l}193 \\
194 \\
195 \\
196\end{array}$ & $\begin{array}{l}\text { TC-90--HD-3 } \\
\text { TC-90-HO-4 } \\
\text { UNDEF INED } \\
\text { UNDEF I NED }\end{array}$ & $\begin{array}{l}336.0 \\
336.0\end{array}$ & $\begin{array}{l}H / L-24 \\
H / L-24\end{array}$ \\
\hline $\begin{array}{l}161 \\
162 \\
163 \\
164\end{array}$ & $\begin{array}{l}\text { SPND-15-1F-S-4 } \\
\text { SPND-15-1A-S-1 } \\
\text { SPND-16-1F-C } \\
\text { UNDEF INEU }\end{array}$ & $\begin{array}{r}97.28 \\
97.28 \\
110.32\end{array}$ & $\begin{array}{l}\text { MT } \\
\text { MT } \\
\text { MT (b) }\end{array}$ & $\begin{array}{l}197 \\
198 \\
199 \\
200\end{array}$ & $\begin{array}{l}\text { PTK-19-3C-C } \\
\text { PTS-19-5C-C } \\
\text { UNDEF INED } \\
\text { UNDEF INEC }\end{array}$ & $\begin{array}{l}157.0 \\
157.0\end{array}$ & $\begin{array}{l}\text { MT } \\
\text { MT }\end{array}$ \\
\hline $\begin{array}{l}165 \\
160 \\
167 \\
168\end{array}$ & $\begin{array}{l}\text { SPNO-17-6A-S-2 } \\
\text { SPND-17-6F-S-3 } \\
\text { SPND-17-1F-S-t } \\
\text { SPND - 17-1A-S-1 }\end{array}$ & $\begin{array}{l}118.28 \\
118.23 \\
118.28 \\
118.28\end{array}$ & $\begin{array}{l}\text { MT } \\
\text { MT } \\
\text { MT } \\
\text { MT }\end{array}$ & $\begin{array}{l}201 \\
202 \\
203 \\
204\end{array}$ & $\begin{array}{l}\text { SRCS-FR-LC-W } \\
\text { SRCS-FR-HI-GH } \\
\text { STBY-FL-OW } \\
\text { U2LP-PR-ES-S-1 }\end{array}$ & $\begin{array}{l}337.0 \\
333.0 \\
382.0 \\
447.0\end{array}$ & $\begin{array}{l}\mathrm{FE}-4(j) \\
\mathrm{FE}-3 \\
\mathrm{FE}-9 \\
\text { PDT-90 }\end{array}$ \\
\hline $\begin{array}{l}169 \\
170 \\
171 \\
i 7 ?\end{array}$ & $\begin{array}{l}\text { UNDEF INED } \\
\text { SPND-18-6F-S-3 } \\
\text { SPND-13-1A-S-1 } \\
\text { UNDEF INED }\end{array}$ & $\begin{array}{l}139.28 \\
139.29\end{array}$ & $\begin{array}{l}\text { (b) } \\
M T_{(b)}\end{array}$ & $\begin{array}{l}206 \\
207 \\
208\end{array}$ & $\begin{array}{l}\text { U2LP-TA-PS-DR-1 } \\
\text { SRCS-S-TC-IN-1 } \\
\text { SRCS-S-TC-OT-1 } \\
\text { SRCS-S-PS-IN-1 }\end{array}$ & $\begin{array}{r}\left\{\begin{array}{r}+447.0 \\
-1176.0\end{array}\right\} \\
-180.0 \\
+363.0 \\
-82.0\end{array}$ & $\begin{array}{l}\text { PDT-90 } \\
\text { TE-2 } \\
\text { TE-3 } \\
\text { PT-5 }\end{array}$ \\
\hline $\begin{array}{l}1 \div 3 \\
174 \\
175 \\
1: 6\end{array}$ & $\begin{array}{l}\text { PS-19-4C-C } \\
\text { PS-19-4C-C-1 } \\
\text { PS-19-3D--C } \\
\text { PS-10-30-C-1 }\end{array}$ & $\begin{array}{l}157.0 \\
157.0 \\
157.0 \\
15 \% .0\end{array}$ & $\begin{array}{l}M T(h) \\
M T \\
M T \\
M T\end{array}$ & $\begin{array}{l}209 \\
210 \\
211 \\
212\end{array}$ & $\begin{array}{l}\text { SRCS-S-PS-OT-1 } \\
\text { SRCS-S-FR-1 } \\
\text { SRCS-S-FR-IN-1 } \\
\text { SRCS-S-FR-DT-1 }\end{array}$ & $\begin{array}{l}+369.0 \\
+950.0 \\
-432.0\end{array}$ & $\begin{array}{l}\text { PT-6 } \\
\text { FY-6 } \\
\text { FV-1 } \\
\text { FI-2(k) }\end{array}$ \\
\hline $\begin{array}{l}17 \\
170 \\
179 \\
180\end{array}$ & $\begin{array}{l}\text { PS-19-4B-C } \\
\text { PS-19-4B-C-1 } \\
\text { PS-19-5D-C } \\
\text { PS-19-5D-C-1 }\end{array}$ & $\begin{array}{l}157.0 \\
157.0 \\
157.0 \\
157.0\end{array}$ & $\begin{array}{l}\text { MT } \\
M i \\
M T \\
M T\end{array}$ & $\begin{array}{l}213 \\
214 \\
215 \\
216\end{array}$ & $\begin{array}{l}\text { SRCS-TC-RF-LP-1 } \\
\text { SRCS-TC-RF-TA-1 } \\
\text { U2LP-FL-OW-1 } \\
\text { UNDEF INEO }\end{array}$ & $\begin{array}{r}-450.0 \\
-225.0 \\
-1560.0\end{array}$ & $\begin{array}{l}\text { TE-17 } \\
\text { TE-18 } \\
\text { FT-40 }\end{array}$ \\
\hline
\end{tabular}




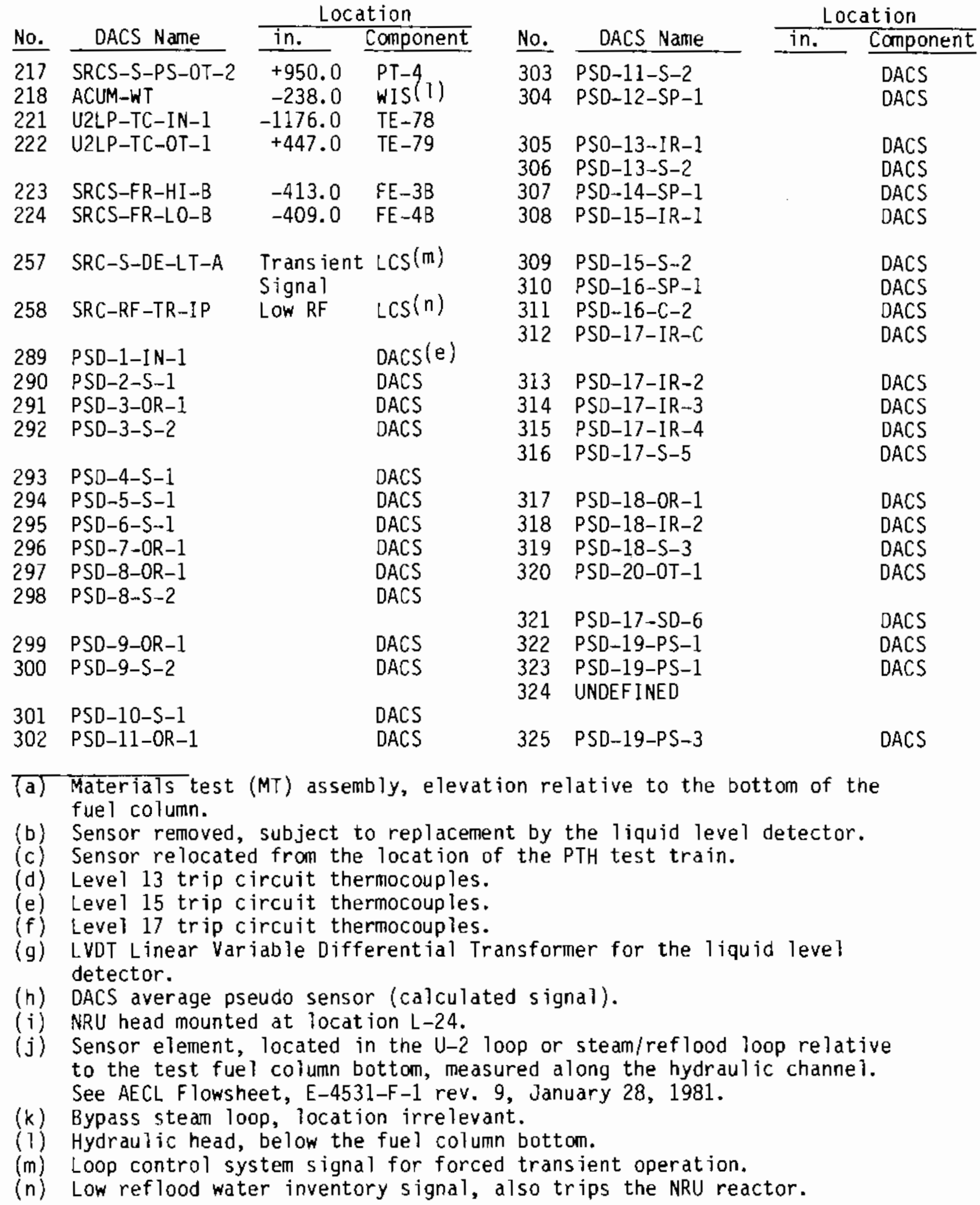


TABLE 3.3. Trip Setpoint Criteria for Operating and Non-Functional

Fuel Cladding Thermocouples

Sensor Instrument Levels

$\frac{\text { Level 17 }}{\text { DACS Thermocouple Numbers }} \frac{\text { Level 15 } 13}{\text { Thermo }}$

Summary Operating Conditions

$2 \leq$ operating TCS on each of levels $13,15,17$

$2 \leq$ Operating TCs on each of levels 15 and 13

$2 \leq$ Operating TCs on each of levels 15 and 17 , or levels 13 and 17

$2 \leq$ Operating TCs on only level 15 or 17 or 13
Criteria and Safety Margins (SM)

Standard $\mathrm{SM}=56 \mathrm{~K}\left(100^{\circ} \mathrm{F}\right)$

Standard $\mathrm{SM}=56 \mathrm{~K}\left(100^{\circ} \mathrm{F}\right)$

Alternate $S M=84 K\left(150^{\circ} \mathrm{F}\right)$

Fal 1-8ack $S M=111 \mathrm{~K}\left(200^{\circ} \mathrm{F}\right)$ $\underline{(99,101,107,109) \quad(98,100,106,108)} \quad(56,57,58,59)$

Trip Setpoint Temperatures

$1333 \mathrm{~K}\left(1940^{\circ} \mathrm{F}\right) \quad 1361 \mathrm{~K}\left(1990^{\circ} \mathrm{F}\right) \quad 1361 \mathrm{~K}\left(1990^{\circ} \mathrm{F}\right)$

$1333 \mathrm{~K}\left(1940^{\circ} \mathrm{F}\right) \quad 1361 \mathrm{~K}\left(1990^{\circ} \mathrm{F}\right) \quad 1361 \mathrm{~K}\left(1990^{\circ} \mathrm{F}\right)$

$1305 \mathrm{~K}\left(1890^{\circ} \mathrm{F}\right) \quad 1333 \mathrm{~K}\left(1940^{\circ} \mathrm{F}\right) \quad 1333 \mathrm{~K}\left(1940^{\circ} \mathrm{F}\right)$

$1305 \mathrm{~K}\left(1890^{\circ} \mathrm{F}\right) \quad 1305 \mathrm{~K}\left(1890^{\circ} \mathrm{F}\right) \quad 1305 \mathrm{~K}\left(1890^{\circ} \mathrm{F}\right)$ 
EHE'ATION, m

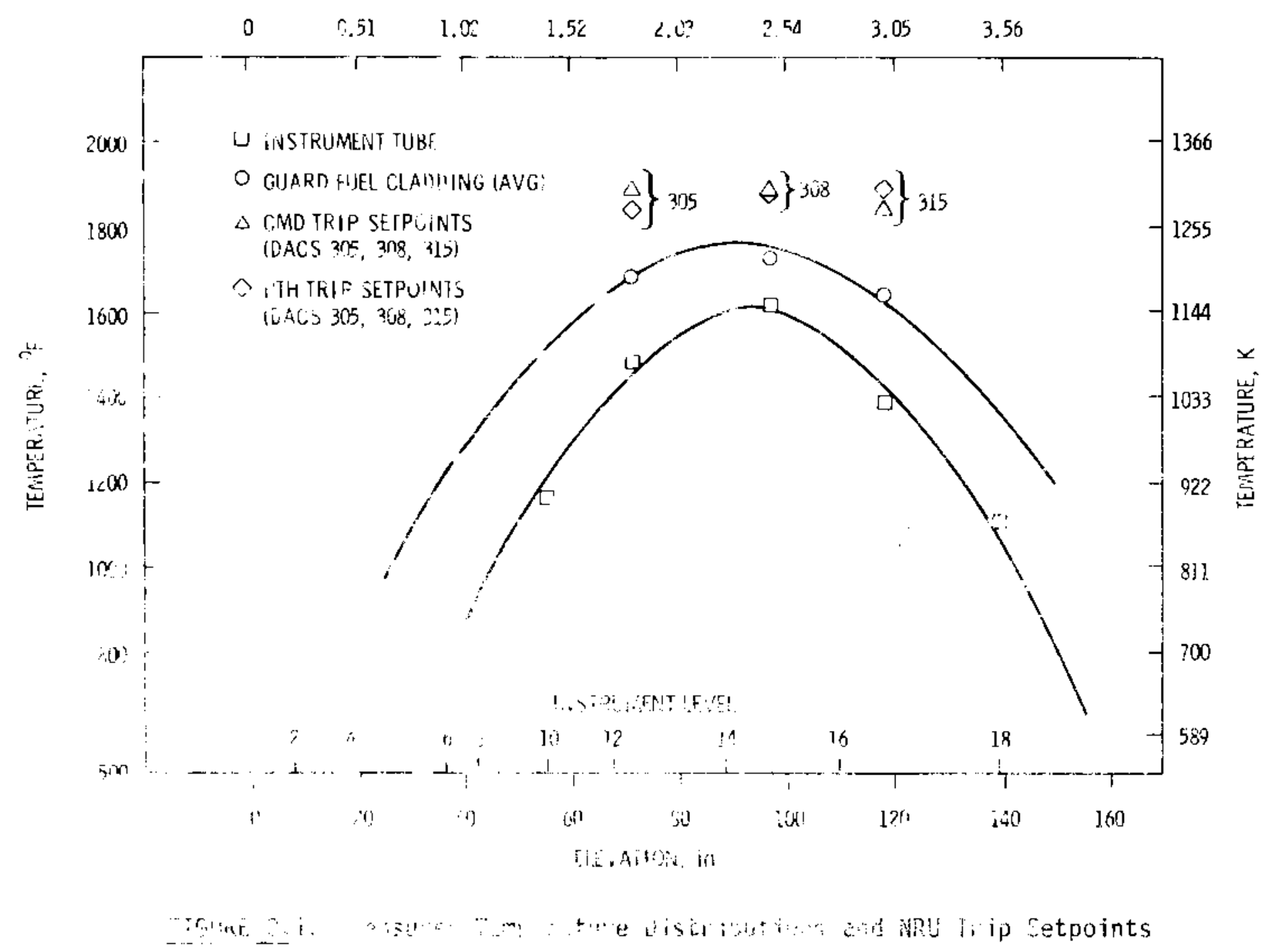


Thermal insulation that protects test instrument connectors at the test train head is composed of several blocks that $f$ it together under the NRU deck plate. These have been redesigned so that they can be removed more quickly without first removing the NRU deck plate. This will minimize personnel exposure to ruptured fuel fission product radiation that may be present in the loop outlet piping under the deck plate.

\subsection{LOOP FACILITIES AND THE LOOP CONTROL SYSTEM}

The steam and reflood Loop Control System (LCS) will be instrumented to provide data to the DACS, essentially the same as provided in the PTH experiment. The only significant change is the location of the steam inlet pressure sensor (PT-5). This is moved as close to the test train inlet as possible (to record steam as well as reflood pressure.) Previously failed inlet and outlet pressure sensors and thermocouples will be replaced and reviewed for operability.

Reflood flow rate data will be provided to DACS for both high (FI-3) and low (FI-4) reflood circuits. Redundant instrumentation will be provided (by sensors $F I-3 B$ and $F I-4 B$ ) to preclude experiment operation without recorded reflood flow rate data. Reflood coolant temperature will also be provided.

Bladders are removed from the reflood water accumulators. This means that nitrogen absorption is not controlled. However, the reflood water in the accumulators will be supplied at the same temperature range, 305-322K $\left(90-120^{\circ} \mathrm{F}\right)$, as it was during the PTH experiment.

In genera1, the LCS wi11 be operated in accord with procedures summarized in section 5 of this addendum. Parameters for both MT experiments are tabulated there. These operations are all similar to the procedures used during the PTH experiment.

\subsection{NRU REACTOR OPERATIONS}

The NRU reactor provides a prototypic power distribution in the test fuel assembly that will restructure the fuel column during the preconditioning phase of the experiment. Fuel pellet/cladding interaction is assured by initially high pressure [8.62 MPa (1250 psia)] coolant, high power density and by fuel 
cracking due to high power operation and the first reactor trip. A schematic of reactor power operations, shown in Figure 3.2, provides an overview of the several test phases for an MT experiment, together with an estimate of the NRU reactor power levels and irradiation times.

Automated NRU power operations for MT-1 are similar to the PTH-110 experiment. Automatic adjuster rod motion is expected during the brief (NRU steady state) period while reflood water is being injected (experiment transient phase). The changing NRU power level during this period will be carefully monitored.

REDACE will be used to collect desired NRU data as rapidly as possible (30s frequency) during the transient phase of each MT experiment. REDACE will record the reactor power, loop operating conditions, platinum SPFD (self powered flux detector) responses, and adjuster rod positions. These magnetic taped data will be used for data reduction analys is and power coupling calculations at PNL after the experiments are completed.

Specific NRU core loading configurations are required for the MT experiments. Selected loads and NRU fuel rods are necessary to provide neutronic conditions similar to the LOCA simulation PJH experiment conditions. These requirements are summarized in Appendix A (M.D. Atfield October 2, 1980). 


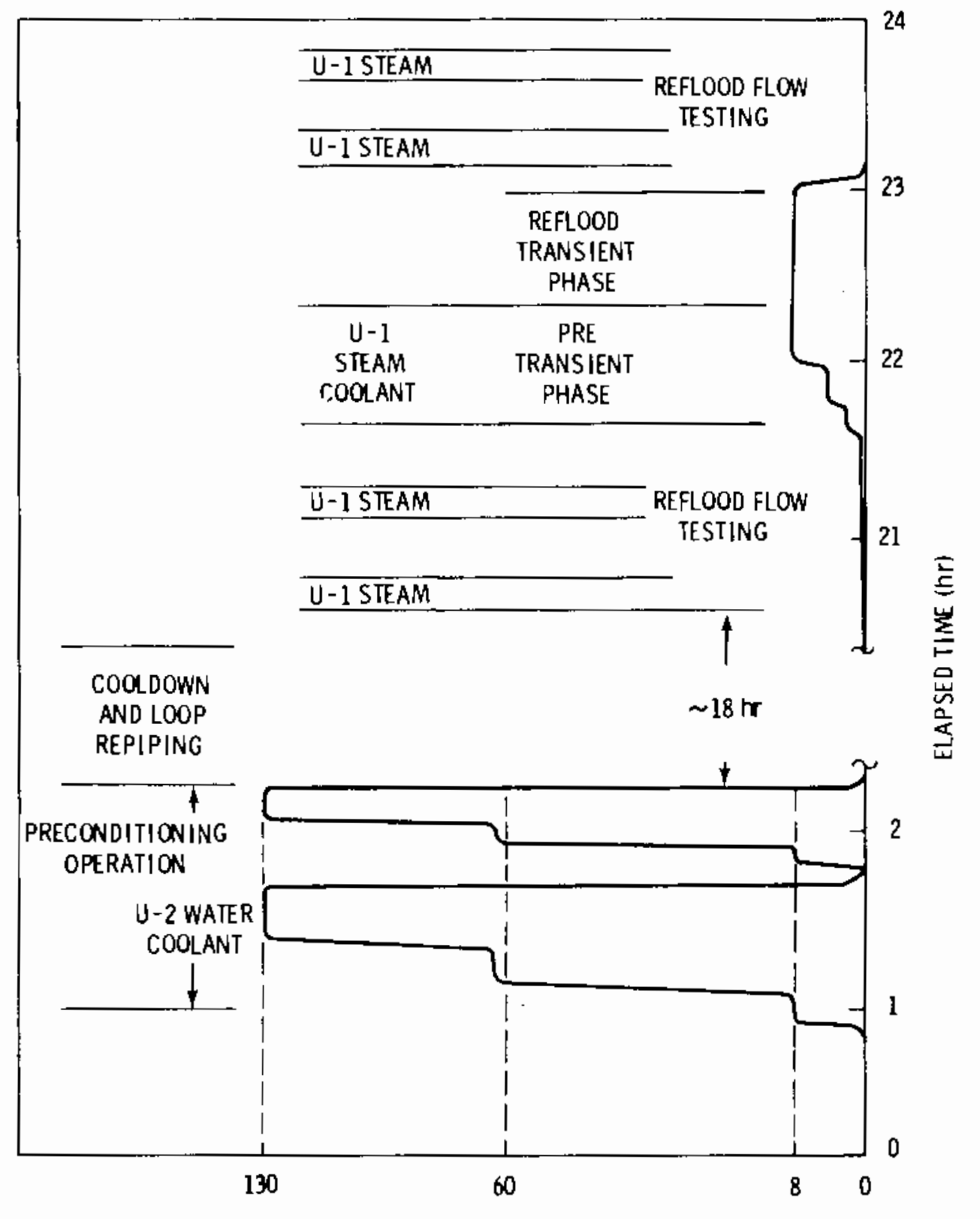

NRU POWER LEVEL (MW)

FIGURE 3.2. MT Experiment and NRU Operations Schematic 


\subsection{HAZAROS REVIEW}

The primary difference between the PTH experiment and these MT experiments is the test fuel rod pressurization change. Of the 31 fuel rods in the test train, the central 11 test fuel rods will be pressurized to $2.66-3.44 \mathrm{MPa}$ (400-500 psia). The maximum internal pressure expected at the time of fue 1 rod rupture is less than $8.28 \mathrm{MPa}(1200 \mathrm{psia})$. At the interior surface of the test train shroud, the stagnation pressure pulse would probably be reduced by at least two orders of magnitude, because the test fuel rods are separated from the shroud by a row of unpressurized guard fuel rods. The NRU pressure tube is fully capable of containing any such pressure pulses caused by fuel rod ruptures due to the fact that it was designed, tested and used for (LOCA simulated) preconditioning test operation at $8.62 \mathrm{MPa}(1250 \mathrm{psia})$. The pressure tube capability is described in the reference report (D.T. Nishimura October, 1980).

Safety analyses, (C. L. Mohr et al. October, 1980) assumed that pressure tube temperatures were as high as $644 \mathrm{~K}\left(700^{\circ} \mathrm{F}\right)$. Throughout the PTH experi- . ment, the maximum temperature measured on the hanger tube was $658 \mathrm{~K}\left(725^{\circ} \mathrm{F}\right)$, but the pressure tube temperature was judged to be at least $50 \mathrm{~K}\left(90^{\circ} \mathrm{F}\right)$ below that. Consequently, the pressure tube design is more than adequate for containing the LOCA simulation MT experiments.

Because the central 11 test fuel rods are expected to rupture during an MT experiment, a summary of the maximum fission product inventory that could be released to the coolant loop and the maximum amount that could be released to the atmosphere are provided in Table 4.1. The calculations were based upon the analyses and data provided in the reference report (D. J. Axford,

I. C. Martin, and S. J. McAuley 1980).

These values are conservative because:

- it is assumed that $1 \%$ of the fission product inventory is released, while considerably less than $1 \%$ release should be expected (I. J. Hastings and M. J. F. Notley 1979, and R. A. Lorenz, D. 0. Hobson, and G. W. Parker 1971); 
Table 4.1. Expected Radioactive Release from

Eleven Test Fuel Rod Ruptures

\begin{tabular}{|c|c|c|c|}
\hline Fission Products & Inventory & Available & Re leasable \\
\hline Equivalent $I^{131}(\mathrm{C} i)$ & 112 & 1.12 & $.011^{(a)}$ \\
\hline Noble Gases (Ci MeV) & 1114 & 11.14 & 11.14 \\
\hline
\end{tabular}
(a) Charcoal filters in the NRU vent line will reduce the released
fission products by $>99 \%$.

- calculated fission product generation was due to a test assembly power level of $2.6 \mathrm{MW}$, compared with $2.0 \mathrm{MW}$, expected to be generated during the preconditioning phase of these experiments; and

- several fission product transport phenomena are also conservatively neglected.

Postulated accidents and the hypothetical fission product releases are discussed in the SAR (C. L. Mohr et al. 1980), and will not be repeated here.

The hydraulic effects of fuel rod rupture have also been considered as a potential hazard of the MT experiments. Simple analyses have shown that at least $90 \%$ coolant channel blockage is required to limit reflood coolant flow by $50 \%$. A nominal $50 \%$ of the desired reflood coolant flow rate was used in analyzing postulated accidents for the SAR. However, even if all (11) test fuel rods rupture (or balloon), substantially blocking all adjacent coolant channels, less than half of the test assembly coolant channel area would be obstructed. This restriction may reduce the reflood flow rate somewhat, but the MT test train will remain adequately coolable under the most conservative postulated blockage conditions. 


\subsection{OPERATING PROCEDURE SUMMARY}

\subsection{PRECONDITIONING OPERATIONS PLAN}

\subsubsection{Piping Configuration}

5.1.1.1 Install test assembly (MT-1) in L-24 test position.

5.1.1.2 Connect U-2 100p to L-24 test position.

5.1.1.3 Pressure test the test train head seal.

\subsubsection{Loop Facilities Preparations}

5.1.2.1 Fill and electrically preneat the U-2 loop.

5.1.2.2 Calibrate loop instruments (see Table 5.1).

5.1.2.3 Confirm trip circuit operability.

5.1.2.4 Implement trip setpoints and report to the experiment director when ready for operation (see Tables 3.3 and 5.2).

\subsubsection{NRU Reactor Preparation}

5.1.3.1 Load NRU reactor fuel assemblies and absorber assemblies, as per Appendix A (M. D. Atfield October 2, 1980).

5.1.3.2 Input NRU reactor linear and log rate trip setpoints, as required by CRNL.

5.1.3.3 Adjust the neutron detector scatter plug, as required.

5.1.3.4 Establish mean power trip setpoints, as required.

5.1.3.5 Confirm that all trip setpoints are activated and report when ready for operation to the experiment director.

\subsubsection{DACS Computer Preparation}

5.1.4.1 Load labeled, certified disk packs and mount a labeled, certified tape on the tape drive (Test Name = MT-1-Pre; Tape No. BTL-035).

5.1.4.2 Start a dummy test and set the DACS to idle mode. 


\section{TABLE 5.1. Loop Instrument Calibration}

\begin{tabular}{|c|c|c|c|c|}
\hline Sensor & _. _oop Paranleter & DACS & - - Instrument Range & Accectaole Accurac \\
\hline \multicolumn{5}{|l|}{ U-2 Loop } \\
\hline $\mathrm{TE}-78$ & Inlet Temperature & 221 & $\begin{array}{l}311 \text { to } 533 \mathrm{~K} \\
170 \text { to } 5000 \mathrm{~F}\end{array}$ & 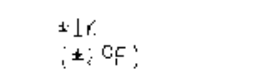 \\
\hline$T E-79$ & Outlet emperature & $2: 2$ & $\begin{array}{l}323 \text { to } 589 \mathrm{~K} \\
\left(122 \text { to } 600^{\circ} \mathrm{F}\right)\end{array}$ & 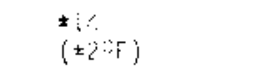 \\
\hline $\mathrm{F} T-40$ & Flow & 215 & $\begin{array}{l}3.9(\mathrm{a}) \text { to } i 9.3(\mathrm{~b}) \mathrm{kg} / \mathrm{s} \\
(36,400 \text { to } 131,400 \mathrm{ibm} / \mathrm{hr})\end{array}$ & $\begin{array}{l}* 0.4 \mathrm{~kg} / \mathrm{s} \\
(+2000 \mathrm{bm} / \mathrm{hr})\end{array}$ \\
\hline POT-90 & Dut let Pressure & 204 & $\begin{array}{l}5.52 \text { to } 3.96 \mathrm{MPa} \\
(800 \text { to } 1300 \mathrm{psid} \text { ) }\end{array}$ & $\begin{array}{l} \pm 0.3 \mathrm{HF} 3 \\
(\$ 50) \mathrm{DS}>0 ;\end{array}$ \\
\hline PDT-90 & Test Assembly Pressure Drop & 205 & $\begin{array}{l}0.021 \text { to } 0.172 \mathrm{MPa} \\
(3 \text { to } 25 \mathrm{psi})\end{array}$ & $\begin{array}{l} \pm ; .4 F^{2} d \\
\{ \pm 0 . \overline{3} \rho \operatorname{si}\}\end{array}$ \\
\hline \multicolumn{5}{|c|}{ Steam/Reflood Loop } \\
\hline$T E-2$ & Inlet Coolant Temperature & 206 & $\begin{array}{l}394 \text { to } 700 \mathrm{~K} \\
\left\{250 \text { to } 300^{\circ} \mathrm{F}\right\}\end{array}$ & $\begin{array}{l} \pm 1 \times \\
\{ \pm 20 F\}\end{array}$ \\
\hline$T E-3$ & Out let Coolant Temperature & 207 & $\begin{array}{l}422 \text { to } 973 \mathrm{~K} \\
\left(300 \text { to } 1300^{\circ}\right)\end{array}$ & $\begin{array}{l}\neq 6 k \\
( \pm 1 j \mathrm{OF})\end{array}$ \\
\hline$F Y-6$ & $\begin{array}{l}\text { Steam Flow Rate, Reflood } \\
\text { Boiling }\end{array}$ & 210 & $\begin{array}{l}0 \text { to } 0.378 \mathrm{~kg} / \mathrm{s} \\
(0 \text { to } 3000 \mathrm{lbm} / \mathrm{hr})\end{array}$ & $\begin{array}{l} \pm 0.014 \mathrm{~kg} / \mathrm{s} \\
( \pm 100 \mathrm{lgm} / \mathrm{hr})\end{array}$ \\
\hline$f \forall-1$ & $\begin{array}{l}\text { Steam System Control flow } \\
\text { Rate, Inlet }\end{array}$ & 211 & $\begin{array}{l}0 \text { to } 0.378 \mathrm{~kg} / \mathrm{s} \\
(0 \text { to } 3000 \mathrm{lbm} / \mathrm{hr})\end{array}$ & $\begin{array}{l} \pm 0.014 \mathrm{~kg} / \mathrm{s} \\
( \pm 100 \mathrm{lgm} / \mathrm{hr})\end{array}$ \\
\hline$F 1-2$ & $\begin{array}{l}\text { Steam System Control Flow } \\
\text { Rate, Outlet }\end{array}$ & 212 & $\begin{array}{l}0 \text { to } 0.032 \mathrm{~kg} / \mathrm{s} \\
0 \text { to } 250 \mathrm{bm} / \mathrm{hr} \text { ) }\end{array}$ & $\begin{array}{l} \pm 0.0013 \mathrm{~kg} / \mathrm{s} \\
\pm 010 \mathrm{~m} / \mathrm{hr})\end{array}$ \\
\hline$P T-5$ & Steam Inlet Pressure & 208 & $\begin{array}{l}0 \text { to } 0.69 \mathrm{MPa} \\
(0 \text { to } 100 \text { psia) }\end{array}$ & $\begin{array}{l} \pm 0.034 \mathrm{MPa} \\
\text { it psidi }\end{array}$ \\
\hline$P \mathbf{T}-6$ & Steam Outlet Pressure & 209 & $\begin{array}{l}0.069 \text { to } 0.345 \mathrm{MPa} \\
(0 \text { to } 50 \mathrm{psia})\end{array}$ & $\begin{array}{l} \pm 0.317 \mathrm{MPd} \\
( \pm 2.5 \mathrm{psid})\end{array}$ \\
\hline $\mathrm{PT}-4$ & $\begin{array}{l}\text { Steam Pressure Control, } \\
\text { Outlet Region }\end{array}$ & 217 & $\begin{array}{l}0 \text { to } 0.69 \mathrm{MPa} \\
\{0 \text { to } 100 \mathrm{ps} \text { ia }\}\end{array}$ & $\begin{array}{l} \pm 0.034: 9 \mathrm{~Pa} a \\
( \pm 5 \text { ps ia) }\end{array}$ \\
\hline$F I-4$ & $\begin{array}{l}\text { Reflood Coolant, Law Flow } \\
\text { Rate }\end{array}$ & 201 & $\begin{array}{l}0.013 \text { to } 0.254 \mathrm{~m} / \mathrm{s} \\
\{0.5 \text { to } 10 \mathrm{in} / \mathrm{s}\}\end{array}$ & $\pm 5:$ \\
\hline$F I-3$ & $\begin{array}{l}\text { Reflood Coolant, High Flow } \\
\text { Rate }\end{array}$ & 202 & $\begin{array}{l}0.013 \text { to } 0.305 \mathrm{~m} / \mathrm{s} \\
(0.5 \text { to } 12 \mathrm{in} / \mathrm{s})\end{array}$ & $\pm 5 \%$ \\
\hline FI40 & $\begin{array}{l}\text { Reflood Coolant, Low Flow } \\
\text { Rate (backup) }\end{array}$ & 224 & $\begin{array}{l}0.013 \text { to } 0.254 \mathrm{~m} / \mathrm{s} \\
(0.5 \text { to } 10 \mathrm{im} / \mathrm{s}\end{array}$ & \pm 5 \\
\hline $\mathrm{FI} 3 \mathrm{~b}$ & $\begin{array}{l}\text { Reflood Coolant, High Flow } \\
\text { Rate (oackup meter) }\end{array}$ & 223 & $\begin{array}{l}0.013 \text { to } 0.305 \mathrm{~m} / \mathrm{s} \\
\{0.5 \text { to } 12 \mathrm{in} / \mathrm{s}\}\end{array}$ & $45 \%$ \\
\hline$F E-9$ & Standby Reflood Coolant Flow & 203 & $\begin{array}{l}0.013 \text { to } 0.305 \mathrm{~m} / \mathrm{s} \\
(0.5 \text { to } 12 \mathrm{in} / \mathrm{s})\end{array}$ & $\pm 10 \%$ \\
\hline $\mathrm{TE}-17$ & $\begin{array}{l}\text { Reflood Coolant Temoerature } \\
\text { Control Valve In let }\end{array}$ & 213 & $\begin{array}{l}305 \text { to } 322 \mathrm{~K} \\
\left(90 \text { to } 120^{\circ} \mathrm{F}\right)\end{array}$ & $\pm 5 \%$ \\
\hline$T E-18$ & $\begin{array}{l}\text { Reflood Coolant Temperature } \\
\text { Control Valve Outlet }\end{array}$ & 214 & $\begin{array}{l}305 \text { to } 322 k \\
(90 \text { to } 120 \%)\end{array}$ & $\pm 5 \%$ \\
\hline
\end{tabular}

(a) At a temperature of $589 \mathrm{~K}\left(600^{\circ} \mathrm{F}\right)$.

(b) At a temperature of $394 \mathrm{~K}\left(2500^{\circ}\right)$. 


\section{TABLE 5.2. Preconditioning Trip Setpoints}

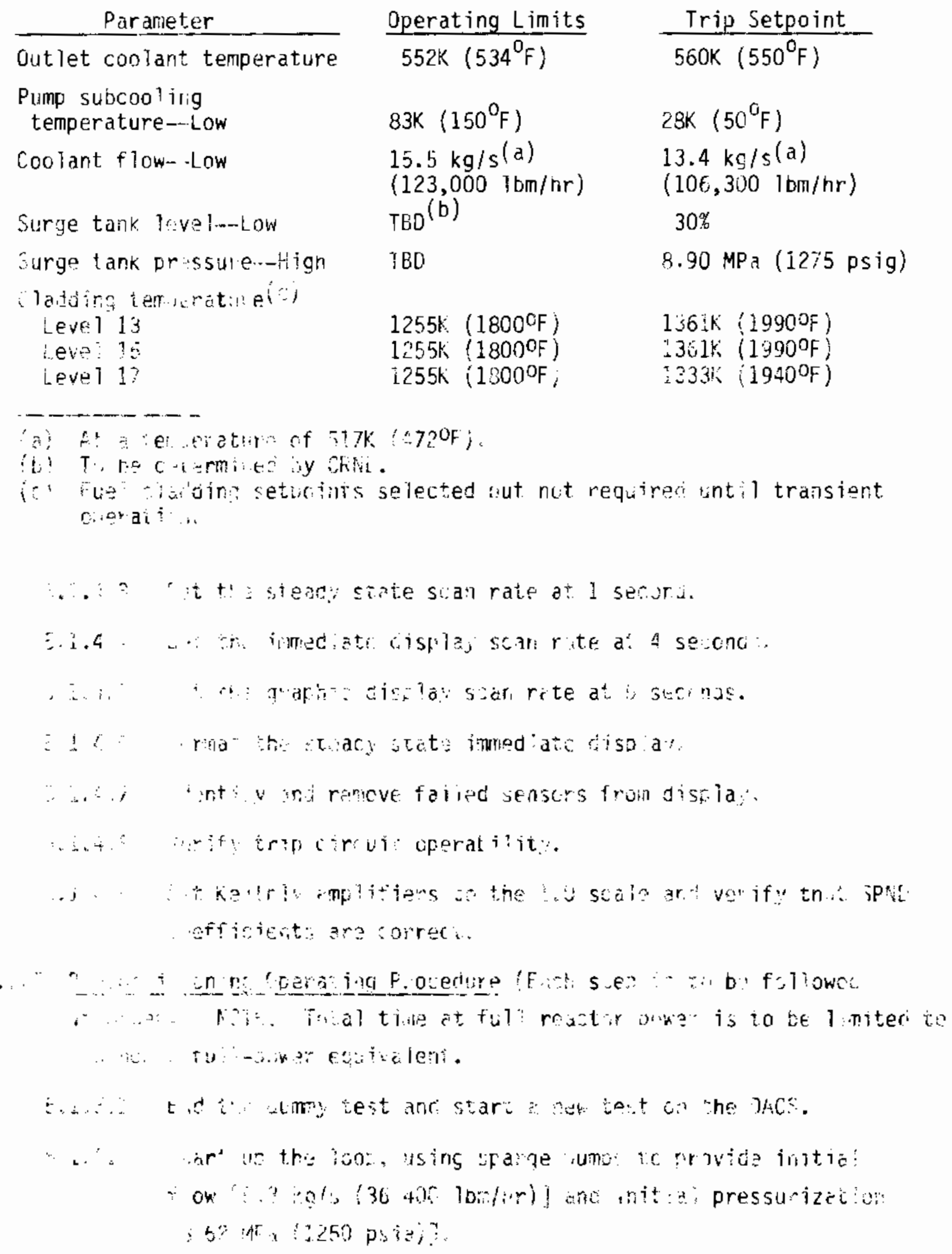


5.1.5.3 Change the DACS mode to steady state.

5.1.5.4 Increase the reactor power to $63.5 \mathrm{MW}$.

5.1.5.5 Perform a power calibration, using a REDACE printout.

5.1.5.6 Print the DACS sensor status report and REDACE printout for review.

5.1.5.7 Increase the U-2 loop flow to $16.3 \mathrm{~kg} / \mathrm{s} \pm 5$

$(129,400 \mathrm{lbm} / \mathrm{hr} \pm 5 \%)$.

5.1.5.8 Increase the reactor power to $127 \mathrm{MW} \pm 5 \%$.

5.1.5.9 Print the DACS sensor status report and the REDACE printout.

5.1.5.10 Perform a power calibration.

5.1.5.11 Conditionally trip the reactor.

5.1.5.12 Using a REDACE print frequency of $30 \mathrm{~s}$, increase the reactor power and U-2 flow rate to full power in 3 steps: $8 \mathrm{MW}, 64 \mathrm{MW}$, $127 \mathrm{MW}=5 \%$, maintaining test assembly inlet temperature at $517 \mathrm{~K} \pm 3 \mathrm{~K}\left(472^{\circ} \mathrm{F} \pm 5^{\circ} \mathrm{F}\right)$, and power skew $<5 \%$.

5.1.5.13 Print the DACS sensor status report.

5.1.5.14 Recheck power calibrations and complete the preconditioning irradiation.

5.1.5.15 Trip the reactor. Turn off the video recorder and record the counter.

5.1.5.16 Make a hardcopy of the CRT immediate display and graphic display showing the hottest centerline thermocouples. Change DACS to idle mode. Make a tape copy when ending the test. Make a disk image copy. Make a historical request for all the data required to run the transient test.

5.1.5.17 Repeat Steps 5.1.5.12 through 5.1.5.16 if time permits.

5.1.5.18 Shutdown the loop facilities to prepare for piping rearrangement. Return DACS to steady state and scan the sensors $1 / m$ in. until reflood flow tests are initiated. 


\subsection{REFLOOD FLOW, PRETRANSIENT AND TRANSIENT OPERATIONS PLAN}

\subsubsection{Piping Configuration}

5.2.1.1 Connect the reflood loop to L-24 test position.

5.2.1.2 Connect the U-1 steam supply to the reflood loop.

\subsubsection{Loop System Preparations}

5.2.2.1 Start up the U-1 loop.

5.2.2.2 Insure that U-2 make up tanks (which supply water to the U-1 loop) are full.

5.2.2.3 Preheat the steam/reflood loop to $408 \mathrm{~K}\left(275^{\circ} \mathrm{F}\right)$.

5.2.2.4 Fill reflood accumulators, at 305-322K (90-120 $\mathrm{F})$. Otherwise drain and refill it.

5.2.2.5 Verify that the supply of nitrogen used for accumulator pressurization is adequate.

5.2.2.6 Calibrate loop instruments, as shown in Table 5.2. Enter conversion factors and units in the test log.

5.2.2.7 Implement the trip setpoints as shown in Tabies 3.3 and 5.4 for the pretransient and transient phases of the experiment. The trip pseudo sensors used on DACS to represent the high cladding temperature trip circuits and sensors are detailed on Table 5.5.

5.2.2.8 Set timer 1, the reflood delay time according to Table 5.6.

5.2.2.9 Set the reflood teminus time (Table 5.6).

5.2.2.10 Set the reflood rate (Table 5.6).

5.2.2.11 Set remaining timers for appropriate reflood durations.

5.2.2.12 Report to the experiment director when ready for experiment operation. 
IAP!5 5.4. Orety ansient Ard Transient Trio Setpoints

\begin{tabular}{|c|c|c|c|c|}
\hline Parameter & Location & Use & $\begin{array}{c}\text { Operating } \\
\text { Limits }\end{array}$ & $\begin{array}{l}\text { Trip } \\
\text { Setpoint }\end{array}$ \\
\hline Hanger Tut: Tompe:ature - High & LCS & $\left\{\begin{array}{l}\text { Pretransient } \\
\text { Transient }\end{array}\right.$ & $691 \mathrm{~K}\left(785^{\circ} \mathrm{F}\right)$ & $839 \mathrm{~K}\left(1050^{\circ} \mathrm{F}\right)$ \\
\hline Outiet Fipe Temperaiure .. High & $\therefore C$ & $\left\{\begin{array}{l}\text { Pretrains ient } \\
\text { Transient }\end{array}\right.$ & $672 \mathrm{~K}\left(750^{\circ} \mathrm{F}\right)$ & $700 K\left(800^{\circ} \mathrm{F}\right)$ \\
\hline 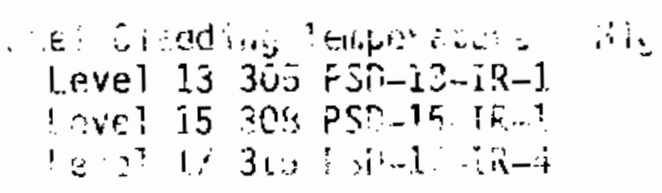 & $\begin{array}{l}\text { DACS } \\
\text { DHAC } \\
\text { Dins: }\end{array}$ & $\begin{array}{l}\text { (Pietransient } \\
\text { |T-ansiant }\end{array}$ & $\begin{array}{l}1305 \mathrm{~K}\left(1890^{\circ} \mathrm{F}\right) \\
1.305 \mathrm{~K}\left(1890^{\circ 5}\right) \\
1 ? 7 \mathrm{SK}(189100 \mathrm{c})\end{array}$ & $\begin{array}{l}1361 \mathrm{~K}\left(1990^{\circ} \mathrm{F}\right) \\
1361 \mathrm{~K}\left(1990^{\circ} \mathrm{F}\right) \\
1333 \mathrm{~K}\left(1940^{\circ} \mathrm{F}\right)\end{array}$ \\
\hline 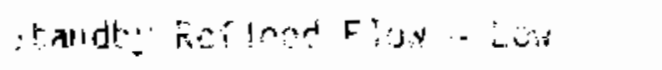 & LIS & Thusisent. & 90 & $.5 \mathrm{in.} / \mathrm{s}$ \\
\hline Rectringletor Inventury -- Luw & 103 & Traisjent & $22.7 \mathrm{~kg}(501 \mathrm{~lm})$ & $11.3 \mathrm{Kg}(25 \mathrm{lbm}$ \\
\hline
\end{tabular}

(a) Standard trip setpoini criteition, see iable 3.3 for nonfunctional $T$, criteria.

th) To he detertined by restl loups steff 
TABLE 5.5. Cladding High Temperature Trip Sensors

\begin{tabular}{|c|c|c|c|}
\hline Leve 1 & $\begin{array}{c}\text { Sensor } \\
\text { Thermocoup les }\end{array}$ & $\begin{array}{c}\text { DACS } \\
\text { Sensor Number }\end{array}$ & $\begin{array}{c}\text { DACS } \\
\text { Pseudo Sensor } \\
\end{array}$ \\
\hline \multirow[t]{4}{*}{13} & TC-13-6C-IR-2 & 56 & 305 PSD-13-IR-1 \\
\hline & TC-13-4F-IR-4 & 57 & 305 PSD-13-IR-1 \\
\hline & TC-13-1D-IR-2 & 58 & 305 PSD-13-IR-1 \\
\hline & $T C-13-3 A-I R-4$ & 59 & 305 PSD-13-IR-1 \\
\hline \multirow[t]{8}{*}{15} & $T C-15-6 C-1 R-4$ & 71 & 308 PSD-15-IR-1 \\
\hline & TC-15-4F-IR-2 & 72 & 308 PSD-15-IR-1 \\
\hline & $T C-15-i D-1 R-4$ & 73 & 308 PSO-15-IR-1 \\
\hline & TC-15-3A-IR-2 & 74 & 308 PSD-15-IR-1 \\
\hline & $T C-15-5 B-I R-3$ & 98 & 308 PSD - 15-IR-1 \\
\hline & $T C-15-2 E-I R-1$ & 100 & 308 PSD-15-IR-1 \\
\hline & $T C-15-5 B-I R-1$ & 106 & 308 PSD-15-IR-1 \\
\hline & TC-15-2E-IR-3 & 108 & 308 PSD-15-IR-1 \\
\hline \multirow[t]{4}{*}{17} & $T C-17-5 E-I R-4$ & 99 & 315 PSD-17-IR-4 \\
\hline & $\mathrm{TC}-17-2 \mathrm{~B}-\mathrm{IR}-2$ & 101 & 315 PSD-17-IR-4 \\
\hline & TC-17-5E-IR-2 & 107 & .315 PSD-17-IR-4 \\
\hline & TC-17-2B-IR-4 & 109 & 315 PSO-17-IR-4 \\
\hline
\end{tabular}

\subsubsection{NRU Reactor Preparations}

5.2.3.1 Confirm that two linear rate and two log rate neutron flux detectors (ion chambers) are being recorded for the experimenter in the NRU reactor control room.

5.2.3.2 Confirm that the REDACE data will be taken on demand or at a 30 s frequency when requested.

5.2.3.3 Establish mean power trip setpoints as required.

5.2.3.4 Confirm that all trip setpoints are activated and report to the experiment director when ready for experiment operation. 
TABLE 5.6. Parameters for Transient and Reflood F low Tests for MT-1 and MT-2

Reflood Tests

\begin{tabular}{|c|c|c|c|c|c|c|}
\hline \multirow{3}{*}{ Test Parameter } & \multirow{2}{*}{\multicolumn{2}{|c|}{ Transient Tests }} & \multirow{2}{*}{\multicolumn{2}{|c|}{ Pre-Transient }} & \multirow{2}{*}{\multicolumn{2}{|c|}{ Post-Transient }} \\
\hline & & & & & & \\
\hline & & $M T-2$ & RT-1 & RT -2 & RT -3 & $R T-4$ \\
\hline Reflood Rate $\mathrm{m} / \mathrm{s}$ ( in/s) & $0.053(2.1)$ & $0.053(2.1)$ & $0.013(.5)$ & $0.53(2.1)$ & $0.013(.5)$ & $0.53(2.1)$ \\
\hline Delay Time (s) & 30 & 12 & 0 & 0 & 0 & 0 \\
\hline $\begin{array}{l}\text { Peak } \delta_{K} \text { ladding Temperature } \\
\left.\qquad{ }^{\prime}\right)\end{array}$ & $\begin{array}{l}1172 \sigma^{K} \\
\left(1650^{F}\right)\end{array}$ & $\begin{array}{l}1061 \gamma^{\gamma} \\
(1450 \mathrm{~F})\end{array}$ & $\begin{array}{l}450 \mathrm{~K} \\
\left(350^{\mathrm{O}} \mathrm{F}\right)\end{array}$ & $\begin{array}{l}450 \mathrm{~K} \\
\left(350^{\mathrm{O}} \mathrm{F}\right)\end{array}$ & $\begin{array}{l}450 g^{k} \\
\left(350^{\circ} F\right)\end{array}$ & $\begin{array}{l}450 \mathrm{~K} \\
\left(350^{\circ} \mathrm{F}\right)\end{array}$ \\
\hline Reflood Teminus Time (s) & 800 & 720 & 165 & 30 & 165 & 30 \\
\hline 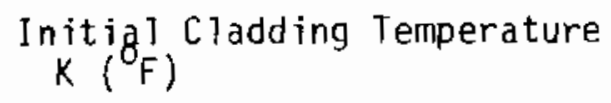 & $\left(8270^{K} \partial_{F}\right)$ & $\begin{array}{l}727 \mathrm{~K} \\
\left(850^{\circ} \mathrm{F}\right)\end{array}$ & $\begin{array}{l}422 \mathrm{~K} \\
\left(300^{\circ} \mathrm{F}\right)\end{array}$ & $\begin{array}{l}422 \mathrm{~K} \\
\left(300^{\mathrm{g}} \mathrm{F}\right)\end{array}$ & ${ }^{422} \mathrm{~K} \mathrm{~J}_{\mathrm{F}}$ & $\begin{array}{l}422 \% \\
(300 \%)\end{array}$ \\
\hline
\end{tabular}




\subsubsection{DACS Computer Preparation}

5.2.4.1 Load the DACS with a labeled certified tape and disks. Test Name MT1-R Tape Number BTL-037

5.2.4.2 Start a dummy test and set the DACS mode to idle.

5.2.4.3 Set the steady state scan rate at 1 second.

5.2.4.4 Set the transient scan rate at $40 \mathrm{~ms}$.

5.2.4.5 Set the immediate display scan rate at 4 seconds.

5.2.4.6 Set the graphic display scan rate at 5 seconds.

5.2.4.7 Format the steady state immediate display.

5.2.4.8 Format the transient graphic display.

5.2.4.9 Identify and remove any failed sensors from displays, pseudo sensors and trip circuits.

5.2.4.10 Reset Keithley amplifiers to the 0.1 scale and change SPND coefficients. Reduce by a factor of 10 .

5.2.4.11 Report to the experiment director when ready for pretransient experiment operation.

\subsubsection{Reflood Flow Test Operating Procedure (To be followed in sequence.)}

5.2.5.1 Calibrate the reflood prefill controls to fill the test nozzle annulus up to level 0 . Prefill and drain.

5.2.5.2 Place the DACS in the steady state mode.

5.2.5.3 Increase test section steam flow to $0.378 \mathrm{~kg} / \mathrm{s}(3000 \mathrm{1bm} / \mathrm{hr})$, and control test section backpressure at $0.276 \mathrm{MPa}$ (40 psia).

5.2.5.4 Enter al1 reflood flow test operational parameters (Table 5.6) in the LCS.

5.2.5.5 Print a sensor status report and ensure that the test assembly thermocouples are $\geq 440 \mathrm{~K}\left(340^{\circ} \mathrm{F}\right)$.

5.2.5.6 Reproduce the CRT immediate display and the graphics display. 
5.2.5.7 Turn on the videotape recorder.

5.2.5.8 Switch the DACS to the transient mode 20 seconds before using the verbal command, "BEGIN THE TRANSIENT," (directed to the LCS operator) and record the time.

5.2.5.9 LCS operator initiates transient.

5.2.5.10 Reproduce the CRT immediate display and the transient graphics display, as required.

5.2.5.11 Stop the test when reflood water passes the thermocouples at level 20.

5.2.5.12 Change the DACS mode to steady state for 5 minutes, then to idle.

5.2.5.13 Make a historical request on the DACS and reproduce copies of the following data:

- reflood rate (DACS sensors 201,202, 203, 223, and 224)

- steam flow-rate (DACS sensors 210, 211, and 212)

- thermocouples at each level (DACS sensors 213, 214, 289, $291,296,297,299,302,304,305,308,313,317$, and 320)

5.2.5.14 Dump all DACS sensors through the transient for each 5 seconds.

5.2.5.15 Check the reflood rate to each leve?

5.2.5.16 Repeat the above steps 5.2.5.1 to 5.2.5.15 using the fast reflood rate. See Table 5.6.

5.2.5.17 Turn off the video recorder and record the counter location.

5.2.5.18 Make a tape copy on the OACS, and make a disk image copy on tape, as time permits.

5.2.5.19 As necessary, repeat DACS Computer preparation (5.2.4) and Loop System Preparation (5.2.2) before proceeding to section 5.2.6, Pretransient Operating Procedure. 


\subsubsection{Pretransient Operating Procedure (To be followed in sequence.)}

5.2.6.1 Start a new test on the DACS. Change the DACS mode to steady state. Test Name $=$ MTl, Tape Number BTL-038

5.2.6.2 Ensure that the REOACE scan frequency for NRU data is 30 seconds.

5.2.6.3 Start up the NRU to the low neutron level.

5.2.6.4 Increase the test section steam flow to $0.378 \mathrm{Kg} / \mathrm{s}(30001 \mathrm{bm} / \mathrm{hr})$ with a 40 psia backpressure.

5.2.6.5 Increase NRU reactor power to 4.0 MW. With the reactor power at nominally $50 \%$ of the pretransient power, Scan the OACS CRT immediate display for the hottest thermocouple and reproduce the display.

5.2.6.6 Increase the steam flow to $0.378 \mathrm{~kg} / \mathrm{s} \pm 5 \%(3000 \mathrm{lbm} / \mathrm{hr} \pm 5 \%)$. Ensure that the test assembly inlet temperature stabilizes at about $450 \mathrm{~K}\left(350^{\circ} \mathrm{F}\right)$ at $4.0 \mathrm{MW}$.

5.2.6.7 Increase the NRU reactor power on automatic control to $\sim 8.0 \mathrm{MW}$.

5.2.6.8 Adjust the reactor power to obtain a steady state peak cladding temperature at $727 \pm 3 \mathrm{~K}\left(850 \pm 5^{\circ} \mathrm{F}\right)$ and outlet steam at about $625 \mathrm{~K}\left(665^{\circ} \mathrm{F}\right)$.

5.2.6.9 Check the peak cladding temperature, steam flow, flux tilt, test assembiy inlet temperature, outlet pressure, and accumulator weight $302 \mathrm{Kg}$ (665 lbs).

5.2.6.10 Reproduce the CRT immediate display and the graphics display.

5.2.6.11 Activate the videotape recorder.

5.2.7 Transient Operating Procedure (To be followed in sequence.)

5.2.7.1 Change to the transient operating mode on the DACS. Twenty seconds later, issue the verbal command, "BEgIN THE TRANSIENT," (directed to the Loop Control 5ystem Operator) and record the time in the DACS rog.

5.2.7.2 The LCS operator begins the transient. 
5.2.7.3 In the DACS $\log$, record the hottest pseudo sensor and the last 2 pseudo sensors to quench.

5.2.7.4 Shut down the reactor when the test assembly quench is complete. 5.2.7.5 Shut off reflood water flow.

5.2.7.6 Record reflood water used (accumulator weight difference).

5.2.7.7 Insure that tripping the reactor has returned control to the DACS (transient forcing signal $\$ 257=0$ ).

5.2.7.8 Return the DACS mode to steady state for 5 minutes. Return the DACS to idle mode, ending the data record.

5.2.7.9 Copy the following historical data on the DACS.

- Make a videotape and hardcopy of the hottest pseudo sensors.

- Make a videotape and hardcopy of the last 2 pseudo sensors to quench.

- Plot the hottest pseudo sensor versus time.

- Plot the last 2 pseudo sensors to quench varsus time.

- Plot loop lata (DACS sensors 201, 20z, 203, 210, 211).

- Dump a 11 cacs data to tape for the transient at 5 second intervais.

5.2.7.10 End the test on the DACS.

5.2.7.11 Turn oft the videotape recorder and record the location.

5.2.7.12 Maxe a tape copy of the DACS data.

5.2.7.13 Make a disc image copy of the CACS dita.

5.2.7.14 Fe:urn io Sectior 5.2.5, Reflcod Flow 0perating Procedure, and fcliow steps 5.2 .5 .1 through 5.2 .5 .18 .

5.2.7.15 Terminate tie experiment and remove the test train to the hay Lising Estaviisned CRNL proceddres. 


\subsection{REFERENCES}

Mohr, C. L., et al. October 1980. Safety Analys is Report: Loss of Coolant Accident Simulations in the National Research Universal Reactor. NUREG /CR-I208, PNL-3093. Pac if ic Northwest Laboratory, Richland, washington.*

Axford, D. J., I. C. Martin, and S. J. McAuley, 1980. Final Safety and Hazards Analysis for the Battelle LOCA Simulation Tests in the NRU Reactor. AECL 7153, Chalk River Nuclear Laboratories. Chalk River, Ontario, Canada.

Russcher, G. E. et al. October 1980. Experiment Operations Plan for a Los5 of Coolant Accident Simulation in the National Research Universal Reactor, NUREG/CR-1735, PNL 3551. Pacif ic Northwest Laboratory, Richland, washington.*

Atfield, M. D., rlemo to NRU Superintendent, Reactor Loading for Battelle LOCA Simulatiors Tests, October 2, 1980. Chalk River Nuclear Laboratories, Chalk River, Oritario, Canada.

Nishimura, [. T. October 1980. Proposal to Install a Zircaloy-2 Pressure whe 450211 for Battelle LOCA Simulation Tests. Exp-Mat-14502, Chalk River Nuclear Laboratories, Chalk River, Ontario, Canada.

Heaberlin, S. w., et al. 1979. Design Basis Neutronics Calculations for NRU LOCA Experinents. PNL-3113, Pacific Northwest Laboratory, Richland, Washington.

Hastings, I. J., and M. J. F. Notley. 1979. Prediction of Fission Gas Re iease from High Burnup 0xide Fuel. AECL-6⿸厂682, Chaik River Nucte ar Laboritories, Chalk River. Ontario, Canada.

Lorenz, R. A., D. O. Hobsor, and G. W. Parker. 1971. Final Report on the First Fuel Rod Failure Transient Test of a Zircaloy-Clad Fuel Rod Cluster in TREAT. OQN!- 4535 , Oak Ridge Nationa Laboratory, Oak Ridge, Tennessee.

*AVollable for puinase from the NRC/GPO Sales Progran, U.S. Nuclear Regulatory Compission, kasiticton, OC 20555 and/or the Nationa! Iechnical Information Service, Springficio, VA 22161. 
APPENDIX A

REACTOR LOADING FOR BATTELLE LOCA SIMULATION TESTS 


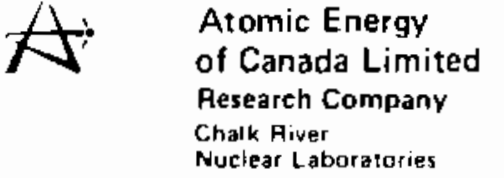

ADVANCED PROJECTS AND REACTOR PHYSICS DIVISION
APPENDIX A du Canada, Limitée Société de Recherehe Laboratoires Nucléaires de Chalk River

TO: NRU Superintendent

FROM: M.D. Atfield

\section{REACTOR LOADING FOR BATTELLE LOCA SIMULATION TESTS}

In order to facilitate the analysis associated with these tests, I would like to maintain the same reactor environment throughout the series. This means that the following sites would contain the specified assemblies any time the BATTELLE fuel is in core:

$$
\begin{aligned}
& \text { J-26 Cobalt carrier rod with very similar loading } \\
& \text { on each occasion. } \\
& \text { K-27 Natural uranium fast neutron rod } \\
& \text { K-21 Aluminum nitride rod } \\
& \text { L-26 FE fuel rod - burn-up } 50 \mathrm{MWD} \\
& \text { M-25 " " " " } 10 \mathrm{MHD} \\
& \text { K-23 " " " " } 130 \text { MWD } \\
& \text { L-22" " " " } 150 \text { MisD }
\end{aligned}
$$

The burn-ups given for driver fuel rod neighbours are approximate and may need to be adjusted some time during the first test to establish more desirable reactor conditions. However, once those rods have been chosen for the first test, they should be preserved for subsequent tests. This would involve extra rod work to install and remove these fuel rods during each BATTELLE shut-down. Storage block space is also required.

Please let me know if this procedure is impractical.

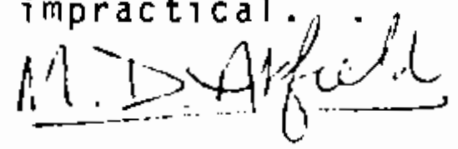

M.D. Atfield

MDA : ny

C.C. C.A. Herriot P.M. Garvey 
NUREG / CR-1735

ADD. -1

PNL-3765

DISTR IBUTION

No. of

Copies

OFFSITE

A. A. Churm

DOE Patent Division

$9800 \mathrm{~S}$. Cass Avenue

Argonne, IL 60439

400 U.S. Nuclear Regulatory Commission

Division of Techical Information and Document Control

7920 Norfolk Avenue

Bethesda, MD 20014

2 DOE Technical Information Center

5 R. Van Houten

U.S. Nuclear Regulatory Commission

Fue] Behavior Branch

Division of Reactor Safety Research

Washington, DC 20555

6 C. A. Herriot

Chalk River National

Laboratory

Chalk River, Ontario,

Canada

ONSITE

33 Pacific Northwest Laboratory

C. L. Mohr

s. 3. Bailey
No. of

Copies

ONSITE

W. D. Bennett

L. W. Cannon

E. L. Courtright

K. E. Davis (6)

M. D. Freshley

R. L. Goodman

C. R. Hann

G. M. Hesson

L. L. King

R. K. Marshall

P. N. McDuffie

C. Nealley

L. J. Parchen

G. E. Russcher (3)

N. J. Wildung

C. L. Wilson

Technical Information (5)

Pub. Coordination (3) 



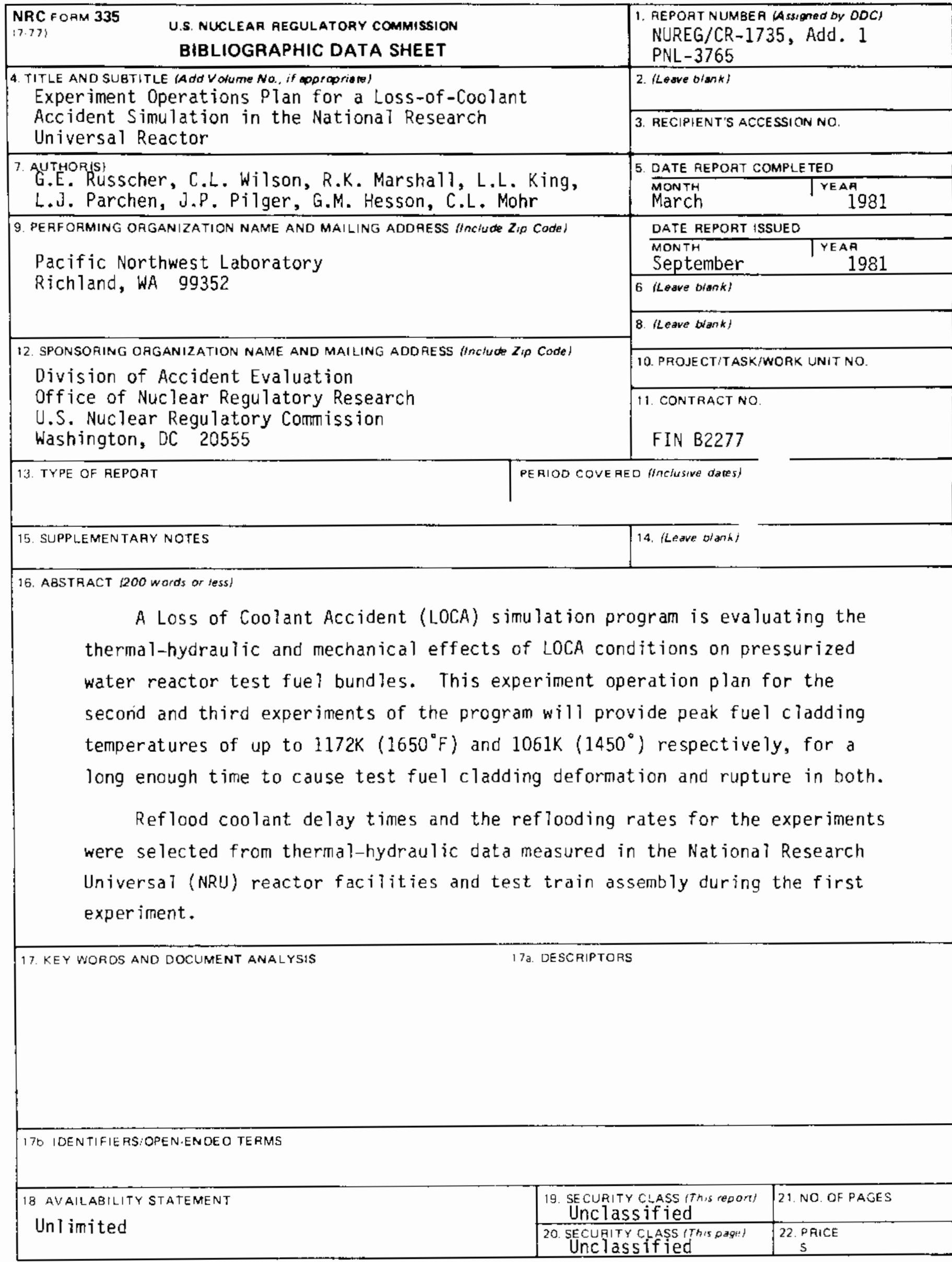


